\title{
Enabling Asynchronous Machine-Type D2D Communication Using Multiple Waveforms in 5G
}

\author{
Conor Sexton, Quentin Bodinier, Arman Farhang, Nicola Marchetti, Faouzi Bader, Luiz A. DaSilva
}

\begin{abstract}
In this paper, we explore the idea that $5 \mathrm{G}$ will permit the use of multiple waveforms, with each service employing a waveform that is best suited for it. We look at a 5G machinetype communication (MTC) scenario consisting of clustered user equipment employing device-to-device (D2D) communication, such as a smart factory with inter-communicating machinery. The overhead associated with synchronising a large number of machine-type D2D user equipment (DUE) comes at a cost that may render synchronous communication infeasible or undesirable. Based on this motivation, we consider multiple possible combinations of prominent 5G waveform candidates for cellular users and DUEs, examining the asynchronous performance of all waveforms under consideration and using the performance of synchronous OFDM as a baseline for comparison. Specifically, we focus on the coexistence of waveforms in which the ordinary cellular users employ OFDM for synchronous communication, as in LTE, and the machine-type DUEs, operating asynchronously, employ a different waveform. When DUEs employ FBMC/OQAM, the average achieved rate is marginally greater than the synchronous OFDM baseline case, and approximately $43 \%$ greater than the asynchronous OFDM case. This result is encouraging, as the benefits of asynchronous D2D communication could be enjoyed in MTC scenarios without suffering any performance reduction compared to the synchronous OFDM scenario. We then investigate how the relative performance of different waveform choices depends on the scenario by varying key parameters. Notably, for asynchronous communication, increasing the transmit power of DUEs results in diminishing benefits unless the DUEs employ a waveform that mitigates inter-device leakage interference.
\end{abstract}

Index Terms-5G, new waveforms, machine-type communication, device-to-device, fractional frequency reuse

\section{INTRODUCTION}

The modulation format and multiple access technique for $5 \mathrm{G}$ are not yet known, with many contenders under consideration, each proving advantageous in certain scenarios and lacking in others. $5 \mathrm{G}$ could potentially be the first generation

Manuscript received XXX, 2017. Revised XXX, 2017. Accepted for publication xxxx, 2018.

Copyright (c) 2017 IEEE. Personal use of this material is permitted. However, permission to use this material for any other purposes must be obtained from the IEEE by sending a request to pubs-permissions @ieee.org.

This publication has emanated from research conducted with the financial support of Science Foundation Ireland (SFI) and is co-funded under the European Regional Development Fund under Grant Number 13/RC/2077. The work of the authors with CentraleSupélec was partially funded through French National Research Agency (ANR) project ACCENT5 with grant agreement code: ANR-14-CE28-0026-02.

Conor Sexton, Nicola Marchetti, and Luiz DaSilva are with the CONNECT Research Centre for Future Networks and Communications, Trinity College Dublin, Ireland. (e-mail: csexton@tcd.ie).

Arman Farhang is with the School of Electrical and Electronic Engineering, University College Dublin, Dublin 4, Ireland.

Quentin Bodinier and Faouzi Bader are with CentraleSuplec/IETR, Rennes 35576, France. to permit the use of different waveforms for different use cases, each one optimal for a given scenario [1]. In this paper, we explore this possibility and focus on the coexistence between two 5G use cases, broadband services and machine-type device-todevice communications, which may use different waveforms.

Many low-power wide-area network solutions, such as NBIoT, have been developed in response to the low rate, latencytolerant traffic that is typically associated with the Internet of Things (IoT). However, there exists a contrasting set of inter-machine communication use cases that will possess requirements for low-latency and potentially high data-rate communication resulting from the increased use of robotics, artificial intelligence, and machine learning across multiple sectors such as energy [2], health, industry, and automotive. In order to enable direct communication between machinetype devices in 5G, D2D communication has been suggested as an enabling technology [3]-[6]. Intelligent process control, autonomous manufacturing systems, smart factories, and selforganising warehouses are but a few examples of $5 \mathrm{G}$ use cases which require direct inter-machine communication in a spatially clustered environment.

The overhead associated with achieving and maintaining synchronous communication for D2D user equipment (DUE) in an MTC scenario can be significant, and it may be desirable to reduce the control burden placed on the base station to achieve this. As highlighted in [7], achieving synchronisation in D2D communications is challenging. This is particularly relevant for clustered machine-type D2D communication scenarios, in which the close proximity of the multiple D2D pairs to each other makes them particularly vulnerable to leakage interference arising from synchronisation errors.

Hence, while ordinary cellular user equipment (CUE) is well served using synchronous communication, clustered machinetype DUEs may instead be best served using asynchronous communication. Clustered MTC, based on D2D communication, is sufficiently different from ordinary cellular traffic to warrant investigation into what waveform choice results in the best performance. Furthermore, the types of devices that we are considering, such as machinery in a smart factory, are designed for specific purposes and may not need to support traditional cellular communication through a base station (BS). In this case, it makes sense for them to use a waveform that is better suited for asynchronous MTC.

Orthogonal frequency division multiplexing (OFDM), employed in LTE, performs quite well when synchronism can be achieved, and some variant of it may continue to be the best choice for cellular communication. However, OFDM's deficiencies in the presence of timing and frequency offsets 
are well known, with several $5 \mathrm{G}$ waveform candidates shown to perform better in asynchronous scenarios [8]. Hence, we are motivated to examine the coexistence of various combinations of waveforms for CUEs and DUEs. We study and quantify how each of the waveforms under consideration performs when employed by DUEs operating in an asynchronous manner, compared to a baseline case consisting of synchronous OFDM. In the remainder of this paper, we use the term alternative waveforms to refer to the multitude of modulation formats that have been proposed in the literature for $5 \mathrm{G}$ as an alternative to OFDM.

In this paper, we expand upon our previous work [9], which demonstrated the effects of inter-D2D interference arising from misaligned communications in a spatially clustered single-cell scenario, and how the use of a waveform that exhibits improved spectral localization over OFDM can mitigate this interference. Here, we expand our work to a multi-cell, multi-cluster network consisting of cells which employ strict fractional frequency reuse (FFR), and consider a wider range of waveforms.

We evaluate the relative asynchronous performance of several waveforms for use in the type of clustered MTC scenario outlined in this section, compared against a baseline case consisting of synchronous OFDM. We also investigate how the level of asynchronism between devices affects the SINR performance of DUEs, examining the effects of both timing offset (TO) and carrier frequency offset (CFO). We stress that our interest lies in evaluating the coexistence and relative performance of different waveforms in such a scenario; we are not concerned with developing a new resource allocation scheme for D2D.

Although waveform research is very mature at a signal processing level, the system level implications and performance of employing different waveforms in different scenarios is not well studied. Our main contribution, therefore, is to demonstrate the benefits that alternative waveforms to OFDM can provide in 5G scenarios such as D2D-enabled MTC. Furthermore, we demonstrate at a system level that waveforms can coexist in 5G, with different devices potentially adopting different waveforms. Our work assesses the performance of a realistic system with a high degree of conformity, without sacrificing realism through simplifying assumptions to obtain tractability.

The main contributions of this paper are the following:

- We compute, and tabulate, the interference arising from the asynchronous coexistence between a large number of alternative waveforms for various timing and frequency offsets.

- We demonstrate using system level simulations that it is feasible for cellular networks to serve high rate clustered MTC use cases using D2D communication through the coexistence of alternative waveforms and OFDM, and quantify the benefit of doing so. In particular, we show that DUEs can avail of the benefits of asynchronous communication, without suffering a performance loss, by employing an alternative waveform such as FBMC/OQAM, even if regular CUEs continue to use OFDM.

- We characterize the performance of several prominent alternative waveforms across a range of MTC scenarios by varying key system parameters such as cell size, cluster size, DUE transmit power, and maximum possible timing offset and CFO.

The rest of the paper is structured as follows. Section II discusses related work, and highlights the novelty of this paper. Section III describes the system model and metrics of interest. Section IV describes the waveforms considered in this paper, and details how leakage interference between pairs of waveforms is modelled. Section V presents and discusses our results, and Section VI concludes the paper.

\section{RELATED WORK}

We build upon our work in [10], which provides interference tables capturing the effects of misaligned D2D users in time onto OFDMA-based cellular users in the uplink band. We also draw upon the work of [11] and [12] in order to characterize the interference imposed between entities utilizing different waveforms.

The use of D2D communication to support MTC scenarios in $5 \mathrm{G}$ appears to be an attractive proposal and has been suggested in several papers [3]-[5]. Our interest in this paper centres around determining the optimal choice of waveform for machine-type D2D communication, and provides a comprehensive analysis of the various options available.

The use of alternative waveforms to OFDM in D2D communications has been considered previously in several papers, and we provide a brief overview of the main works here. In [13], the authors investigate a D2D video transmission network in which D2D transceivers use filtered multi-tone (FMT) in order to ensure that no inter-carrier interference occurs. In [14], the authors consider power loading for D2D pairs operating in an asynchronous manner, and compare the performance of OFDM and filter bank multi-carrier with offset-QAM (FBMC/OQAM). However, no cellular users are considered in either of the above works and therefore issues regarding the coexistence of waveforms for different types of users do not arise.

The authors in [15] aim to maximise the sum-rate for asynchronous D2D underlay communications and consider the use of both FBMC and OFDM. [16] investigates resource sharing between D2D and cellular users in the downlink band, and suggests the use of filtered-OFDM (f-OFDM) for D2D devices to enable them to use parts of the guard band. In [17], the authors study the use of universal filtered OFDM (UFOFDM) for D2D communication, but focus solely on interD2D interference between D2D pairs that are not in the same cell. D2D pairs are assumed to synchronize to CUEs only if they are in the same cell in [18].

Our work differs from the aforementioned papers in many regards. Firstly, we are concerned with asynchronous direct communication whereby each DUE cannot be assumed to be synchronised with any other device in the system. We are also targeting the use of direct communication in spatially clustered MTC applications in which the inter-device leakage interference arising from misaligned communication plays a key role. The key novelty of this work lies in its comparison 
of the performance of multiple waveforms, whereby the waveform used by DUEs and CUEs may be different. Finally, we also consider a multi-cell system employing a frequency reuse technique known as strict FFR (outlined in Section III), and consider all possible interference links in the system in order to obtain realistic results. To the best of our knowledge, no work available in the literature tackles inter-user interference caused by the asynchronous coexistence of multiple use cases employing different waveforms with a similar level of detail and for so many different waveforms.

We highlight that several works [19]-[21] have already investigated direct communication in FFR systems; however, they are not concerned with the use of alternative waveforms in asynchronous direct communication between devices. We include the use of strict FFR in this work to achieve a higher level of realism in our analysis, as it is a frequently employed technique in modern networks to tackle inter-cell interference.

\section{System Model}

We expand upon the system model presented in our previous work [9], where we investigated the performance of D2D communication underlaying an OFDMA based network in the uplink for a single cell scenario.

\section{A. Network Set-up}

We consider an OFDMA network with parameters selected based on the 3GPP LTE standard, as outlined in Table II in Subsection V-A. Cells are modelled as hexagons, with the network consisting of a central cell of interest, an inner ring of direct neighbour cells, and an outer ring of additional cells (totalling nineteen cells). Two rings of cells are commonly used with the hexagonal cellular model, as interference from cells outside of this range can generally be considered negligible. Indeed, we verified this using our simulator, confirming that the addition of a third ring of cells has a negligible influence on the results.

We assume that each cell is fully loaded, with each CUE assigned a single uplink RB. DUEs coexist with the OFDMA cell by reusing a single uplink resource block (RB). In reality, LTE networks actually use single-carrier FDMA (SC-FDMA) in the uplink. However, SC-FDMA is simply OFDMA in which users apply DFT precoding to their transmit signal. In the scope of our study, this precoding is inconsequential and we therefore do not consider it.

CUEs are distributed throughout the entire network according to a Poisson point process (PPP). DUEs are employed in high-rate spatially clustered applications such as process control, robotics control, or machine-to-machine communications. In order to capture this clustering effect in our model, we distribute DUE transmitters in the network using a Matérn point process. For each DUE transmitter, we distribute a receiver at a distance $d$ according to a uniform random variable $U_{[a, b]}$, with $a$ and $b$ representing the minimum and maximum distance, respectively.

As stated in Section I, we consider the use of strict fractional frequency reuse. Fig. 1 illustrates the division of sub-bands between cells. The CUEs in the inner region of each cell

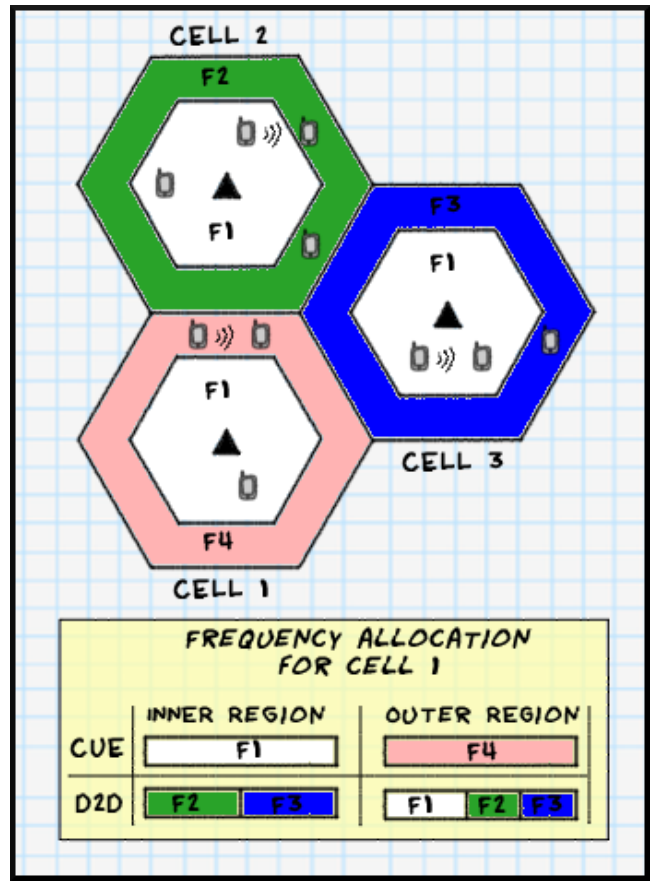

Fig. 1. The inner region of each cell uses the same set of sub-bands, while reuse three is employed in the outer regions. CUEs and DUEs are allocated sub-bands in a manner that aims to reduce interference between them, according to the scheme outlined in [19].

are provisioned using a common set of sub-bands. Frequency reuse three is employed for the outer regions of the cells, with cell-edge CUEs in these regions provisioned from one of three sets of sub-bands. Machine-type DUEs are permitted to reuse the spectral resources of cellular users according to the scheme outlined in [19] for D2D communication operating in a network employing strict FFR. Hence, DUEs in the inner region of a cell may reuse the spectral resources assigned to CUEs in the outer regions of neighbouring cells ${ }^{1}$. DUEs in the outer region of a cell may use any spectral resource, except the set assigned to CUEs in the same region.

The scenario under evaluation in this paper is similar to underlay, since neither D2D devices nor cellular users have exclusive use of the available spectrum across the entire network. However, we also note that due to the manner in which sub-bands are assigned through the use of fractional frequency control, cellular users and D2D devices do not use the same spectral resources within the same region. Hence, the system could be described as overlay at a local level and underlay at a system-wide level, and does not conform to the strict definition of either term.

The ratio of the radius of the inner region $\left(R_{\text {inner }}\right)$ to the radius of the cell $\left(R_{\text {cell }}\right)$ is an important parameter in strict FFR systems and influences how sub-bands are divided between regions. We follow the approach used in [22], and choose

\footnotetext{
${ }^{1}$ Note that DUEs in the inner region of a cell may not reuse the resources of cellular users in the outer region of the same cell, as the DUEs would always be closer to the base station than the CUEs. Since interference in the uplink occurs at the base station, this could result in significant interference from DUEs to CUEs, which is precisely what the reuse scheme is designed to prevent.
} 
the ratio $R_{\text {inner }} / R_{\text {cell }}$ to be 0.65 , which was shown in [23] to maximise the average network throughput for uniformly distributed CUEs. Given $N_{\text {band }}$ available sub-bands in the system, we can determine the number of resources allocated to each region as follows [23]

$$
\begin{aligned}
& N_{\text {inner }}=\left[N_{\text {band }}\left(\frac{R_{\text {inner }}}{R_{\text {cell }}}\right)^{2}\right], \\
& N_{\text {outer }}=\left[\left(N_{\text {band }}-N_{\text {inner }}\right) / 3\right] .
\end{aligned}
$$

\section{B. Resource Allocation}

RBs are assigned under the condition that an RB may only be assigned to a single CUE, and reused by a single DUE, in a given cell. CUEs transmit on the physical uplink shared channel (PUSCH) and use a power control procedure [24] that assigns each CUE a power level that results in acceptable signal reception at the base station. In the procedure used, the pathloss between each CUE and the BS is estimated and compensated for in order to satisfy the power that the base station expects to receive over a single resource block $P_{\text {O_PUSCH}}$. The maximum power at which a CUE may transmit is also capped at $P_{\text {cmax }}$.

Our focus in this paper is on evaluating the relative performance of the waveforms under consideration for direct communication between devices/equipment in spatially clustered use cases, not on proposing a new resource allocation scheme. Hence, in order to avoid bias towards any particular scheme, we consider a simple power allocation scheme for machine-type DUEs whereby they are permitted to transmit at maximum power, which is capped by the controlling base station.

The results section provides detailed insight into the performance of asynchronous communication for various waveforms, compared to a baseline of synchronous communication, allowing informed decisions to be made regarding the choice of waveform for both types of communication. We highlight, however, that the decision of whether to use synchronous or asynchronous communication is multifaceted and there are many reasons why an operator may decide to employ asynchronous communication for MTC scenarios. As mentioned, the cost of achieving and maintaining synchronous communication for MTC may be unattractive. Removing the synchronisation procedure for DUEs could help to reduce the latency experienced by these devices. Asynchronous DUE communication also removes several duties of control from the base station, potentially enabling the network operator to treat resource allocation for high-rate clustered MTC scenarios in a different manner than for CUEs. For example, the network could release spectral resources to a smart factory without actively managing the directly communicating machinery, which may operate autonomously or via a local controller.

\section{Channel Modelling}

CUEs in the same cell do not interfere with each other, as we assume they are perfectly synchronized by the BS. Therefore, there are four main interference types requiring consideration:
1) DUE pairs interfere with the CUEs' transmissions. Since we are investigating uplink resource sharing, this interference is observed at base stations.

2) Conversely, the CUEs interfere with the DUE pairs at DUE receivers.

3) DUEs interfere with each other (inter-DUE interference).

4) CUEs in different cells are not synchronized and, hence, interfere with each other (inter-CUE interference).

Owing to their popularity in the existing literature [18], [25], [26], we employ the WINNER II channel models [27] to provide us with a distance based path loss, which also incorporates the probability of line-of-sight. Distinct path loss models are used for the different types of links in the system in order to represent the network in a realistic manner. Path loss models employed for D2D channels have been modified so that both transceivers in a D2D link are the same height above the ground. The distribution of shadow fading is lognormal, with the standard deviation specified by the Winner II channel models for each scenario.

\section{Performance Measures}

Below, we present several metrics that we will use to evaluate the performance of the system. All metrics are evaluated for DUEs and CUEs in the central cell, which represents the cell of interest.

1) SINR: The SINR of a CUE $j$ in the central cell $o$ using $\mathrm{RB} k$ is given by:

$$
\gamma_{j_{o}}^{k}=\frac{P_{j_{o}}^{k} h_{j_{o} B}^{k}}{\sigma_{\nu}^{2}+I_{\mathrm{C}_{\mathrm{N}}}+I_{\mathrm{D}_{\mathrm{N}}}+I_{\mathrm{D}_{\mathrm{S}}}}
$$

where $P_{j_{o}}^{k}$ is the transmit power of the CUE, $h_{j_{o} B}$ is the channel gain between the $j^{\text {th }}$ CUE and the BS of the central cell $o$, and $\sigma_{\nu}^{2}$ is additive white Gaussian noise variance. $I_{\mathrm{C}_{\mathrm{N}}}$ is the interference from CUEs in neighbouring cells and is given by

$$
I_{\mathrm{C}_{\mathrm{N}}}=\sum_{n \in N} \sum_{c_{n} \in C_{n}} \sum_{r \in R} P_{c_{n}}^{r} h_{c_{n} B}^{r} \Omega_{\mathrm{wf}_{c_{n}} \rightarrow \mathrm{wf}_{j_{o}}}\left(|r-k|, \delta_{\mathrm{t}}, \delta_{\mathrm{f}}\right),
$$

where $n$ indexes the set of neighbouring cells $N, c_{n}$ indexes the CUEs in the set $C_{n}$ of CUEs in the $n^{\text {th }}$ neighbouring cell, and $r$ indexes the set of resource blocks $R$ available to the system. $P_{c_{n}}^{r}$ is the transmit power of the $c_{n}^{\text {th }}$ CUE operating on RB $r, h_{c_{n} B}^{r}$ is the channel gain between the $c_{n}^{\text {th }}$ CUE and the BS of the central cell. If the $c_{n}^{\text {th }}$ CUE is not operating on RB $r$, then $P_{c_{n}}^{r}$ is 0. Finally, $\Omega_{\mathrm{wf}_{c_{n}} \rightarrow \mathrm{wf}_{j_{o}}}\left(|r-k|, \delta_{\mathrm{t}}, \delta_{\mathrm{f}}\right)$ is the fraction of power injected by CUE $c_{n}$ using waveform $\mathrm{wf}_{c_{n}}$ and resource block $r$ onto CUE $j_{o}$ using waveform $\operatorname{wf}_{j_{o}}$ and resource block $k$, at a timing offset of $\delta_{\mathrm{t}}$ and CFO $\delta_{\mathrm{f}}$. For synchronous communication, both $\delta_{\mathrm{t}}$ and $\delta_{\mathrm{f}}$ can be set to 0 . $I_{\mathrm{D}_{\mathrm{N}}}$ is the interference from DUEs in the neighbouring cells and is given by

$$
I_{\mathrm{D}_{\mathrm{N}}}=\sum_{n \in N} \sum_{d \in D_{n}} \sum_{r \in R} P_{\mathrm{D}} h_{d_{n} B}^{r} \Omega_{\mathrm{wf}_{d_{n}} \rightarrow \mathrm{wf}_{j_{o}}}\left(|r-k|, \delta_{\mathrm{t}}, \delta_{\mathrm{f}}\right),
$$

which is defined in a similar fashion to Eq. (4), where $D_{n}$ represents the set of DUEs in the $n^{\text {th }}$ neighbouring cell, and $P_{\mathrm{D}}$ is the transmit power of DUE devices. Finally, $I_{\mathrm{Ds}}$ 
represents the interference from DUEs in the same cell, i.e. the central cell, and is formulated in a similar fashion to Eq. (5).

The SINR of a DUE $d$ in the central cell $o$ operating on $\mathrm{RB} r$ is given by:

$$
\gamma_{d_{o}}^{r}=\frac{P_{\mathrm{D}} h_{d_{o}}^{r}}{\sigma_{\nu}^{2}+I_{\mathrm{C}_{\mathrm{N}}}+I_{\mathrm{C}_{\mathrm{S}}}+I_{\mathrm{D}_{\mathrm{S}}}+I_{\mathrm{D}_{\mathrm{N}}}},
$$

where $P_{\mathrm{D}}$ is the transmit power of the DUE devices, $h_{d_{o}}^{r}$ is the channel gain between the transmitter and receiver of the $d^{\text {th }}$ DUE using RB $r$, and $\sigma_{\nu}^{2}$ is additive white Gaussian noise variance. $I_{\mathrm{C}_{\mathrm{S}}}$ and $I_{\mathrm{C}_{\mathrm{N}}}$ represent the aggregate interference from CUEs in the same cell and neighbouring cells, respectively. $I_{\mathrm{D}_{\mathrm{S}}}$ and $I_{\mathrm{D}_{\mathrm{N}}}$ represent the aggregate interference from DUEs in the same cell and neighbouring cells, respectively. The expressions for each of the above aggregate interference terms are similar to Eqs. (4) and (5), with the channel gain $h$ considered between the interfering device and the DUE receiver.

2) Achieved Rate: We are also interested in the rate achieved by devices, after the bandwidth efficiency of each waveform has been taken into account. The rate of a device using a waveform wf can be calculated as

$$
b=\Phi_{\mathrm{wf}} B \log _{2}(1+\gamma)[b / s],
$$

where $B$ is the bandwidth of an LTE resource block, and $\Phi_{\mathrm{wf}}$ is the bandwidth efficiency of waveform wf presented in Table I, which is directly computable based on the waveform parameters presented in the same table.

\section{CANDidate WaVeforms And Leakage INTERFERENCE MODEL}

\section{A. Candidate Waveforms under Study}

Below, we briefly present the waveforms that we will consider in this paper.

1) Orthogonal frequency division multiplexing (OFDM): $\mathrm{Al}-$ though alternative waveforms are being studied, OFDM may still have an important role to play in 5G. OFDM works quite well in the downlink of cellular systems. In scenarios that do not comprise machine type communication or delay-intolerant communications, the signalling overhead required to align individual devices becomes affordable. Moreover, MIMO techniques, a key part of future $5 \mathrm{G}$ systems, are directly applicable to OFDM.

However, OFDM suffers from high out-of-band emissions and is known to perform poorly in situations where multiple users transmit adjacently and asynchronously, which is precisely the class of network deployments that interests us in this study.

2) Filter bank multi-carrier (FBMC): FBMC waveforms apply an enhanced filtering on a per-subcarrier level in order to remove the large sidelobes typically associated with OFDM transmission. This filtering makes FBMC subcarriers highly spectrally localized, which reduces the sensitivity to asynchronism. Moreover, FBMC systems generally do not rely on a cyclic prefix (CP) to combat inter-symbol interference (ISI). The combination of these two attributes, reduced sidelobes and no $\mathrm{CP}$, results in a time-frequency efficiency that is very close to 1 (and approaching 1 in the ideal case of infinite block lengths). Instead, the very narrow channels used in FBMC guarantee flat gain. However, it should be noted that in highly frequency selective channels, existing channel estimation methods for FBMC without a CP cannot fully remove ISI, with performance suffering as a result. Hence, CPbased FBMC systems have been investigated [29], [30] to fully remove ISI in frequency selective channels and achieve simple one-tap equalization similar to OFDM.

Several FBMC-based schemes have been proposed in the literature. In this paper, we study the following ones:

- Filtered Multi-Tone (FMT) [31]: to reduce out-of-band emission, every subcarrier in FMT is filtered by a narrow passband filter, which inevitably results in the loss of the orthogonality between subcarriers according to the Balian-Low theorem. To deal with this, a guard band is added between every subcarrier; however, this reduces the spectral efficiency of the system.

- FBMC/OQAM [32], [33]: possibly the most wellknown alternative to OFDM, FBMC/OQAM achieves maximum spectral efficiency by removing the guard bands used in FMT. Inter-carrier interference (ICI) and ISI are eliminated by using OQAM modulation instead of QAM. However, FBMC/OQAM systems achieve orthogonality only in the real domain, and suffer from pure imaginary interference which can be detrimental for equalization and makes their application to MIMO challenging.

- FBMC-PAM (also known as lapped FBMC) [34]: whereas FBMC/OQAM systems double the symbol rate, FBMC/PAM doubles the number of subcarriers. It also uses a short sine filter which achieves a good trade-off between time and frequency localization.

3) Generalised frequency division multiplexing (GFDM) [35]: One of the main drawbacks of the aforementioned FBMC waveforms is the delay incurred by linear convolution with the prototype filter on each subcarrier. To overcome this issue, GFDM applies circular convolution to filter independent data blocks consisting of $K$ subcarriers and $M$ sub-symbols per subcarrier. In addition, only one cyclic prefix is applied per entire block in order to reduce the block overhead. However, circular filtering results in non-orthogonal subcarriers, introducing both ISI and ICI which must be dealt with using interference cancellation techniques, or linear decoders which increase the error rate and complexity of the receiver compared to OFDM. Note that some works have studied the joint use of OQAM and GFDM and proposed circular OQAM (COQAM) waveforms [36]. However, it has been shown that GFDM and COQAM achieve comparable performance in terms of asynchronous coexistence [37]. This leads us to leave COQAM out of the scope of our study and to consider GFDM only as an example of a circularly pulse-shaped waveform.

4) Universal filtered multi-carrier (UFMC) and filtered- 
TABLE I

PARAMETERS OF CONSIDERED WAVEFORMS

\begin{tabular}{|c|c|c|c|c|c|c|c|c|}
\hline Parameter & Symbol & OFDM & FMT & $\begin{array}{c}\text { FBMC / } \\
\text { OQAM }\end{array}$ & $\begin{array}{c}\text { FBMC- } \\
\text { PAM }\end{array}$ & GFDM & f-OFDM & UFMC \\
\hline Time-symbol & $\mathrm{T}$ & $\frac{1}{\Delta \mathrm{F}}$ & $\frac{1}{\Delta \mathrm{F}-W_{\mathrm{GB}}}$ & \multicolumn{5}{|c|}{$\frac{1}{\Delta \mathrm{F}}$} \\
\hline Cyclic Prefix & $T_{\mathrm{CP}}$ & $\frac{T}{8}$ & & & & \multicolumn{3}{|c|}{8} \\
\hline Filter & & & $\begin{array}{l}\text { root raised } \\
\text { cosine } \\
\text { (RRC), } \\
\text { rolloff } \\
0.22, \\
\text { duration } \\
6 T\end{array}$ & $\begin{array}{c}\text { Phydyas, } \\
\text { duration } \\
4 T\end{array}$ & $\begin{array}{l}\text { Sine filter, } \\
\text { duration } \\
2 T\end{array}$ & $\begin{array}{l}\text { RRC, } \\
\text { rolloff 1, } \\
\text { duration } \\
5 T\end{array}$ & $\begin{array}{l}\text { Truncated } \\
\text { sinc filter } \\
{[28] \text { with }} \\
T_{w}=\frac{T}{2}\end{array}$ & $\begin{array}{c}\text { Chebyshev, } \\
-60 \mathrm{~dB} \\
\text { attenua- } \\
\text { tion, } \\
\text { duration } \\
T_{\mathrm{CP}}\end{array}$ \\
\hline Active subcarriers per resource block & & \multicolumn{2}{|c|}{12} & \multicolumn{2}{|c|}{11} & 12 & 11 & 12 \\
\hline Bandwidth efficiency & $\Phi_{\mathrm{wf}}$ & $8 / 9$ & $8 / 9$ & \multicolumn{2}{|c|}{$11 / 12$} & $\begin{array}{c}5 /(5+ \\
1 / 8)\end{array}$ & $\begin{array}{l}8 / 9 * \\
11 / 12\end{array}$ & $8 / 9$ \\
\hline
\end{tabular}

OFDM (f-OFDM): UFMC [38], also known as UFOFDM, aims to generalize OFDM and FBMC in order to reap the benefits of both while avoiding their respective limitations. While FBMC filters individual subcarriers, UFMC applies filtering to groups of adjacent subcarriers, which reduces the ramp-up and down delays of the prototype filter. One of the advantages of UFMC lies in the fact that it preserves time orthogonality between subsequent symbols by limiting the filter tails within the guard interval. However, this does not allow for long filters and may therefore make it difficult to achieve satisfactory out-of-band rejection levels when dealing with signals that have a small passband. To overcome this, f-OFDM systems [28] use longer filters, which enable communication devices to achieve sharper frequency localization at the cost of orthogonality between subsequent symbols.

\section{B. Implementation Parameters Selected for Waveforms under Study}

All of the aforementioned waveforms have fostered a wide range of works [39], [40], with a large number of different implementations and parameters considered in the literature. Studying all the proposed variations of these waveforms would therefore be infeasible. Hence, to ensure fair comparison, we choose parameters, filters, and implementations that are representative of most works in the literature.

As our work focuses primarily on investigating how alternative waveforms can facilitate coexistence in a certain band of the wireless spectrum, we consider that each studied modulation scheme uses the same subcarrier spacing $\Delta \mathrm{F}=15 \mathrm{kHz}$ in accordance to current LTE standards to ensure fairness in our comparisons. Note that, in the particular case of FBMC-PAM, each subcarrier is actually composed of two virtual subcarriers of width $\Delta \mathrm{F} / 2$. Other relevant parameters are presented in Table I. $T$ represents the time symbol, $T_{\mathrm{CP}}$ is the cyclic prefix or guard interval duration where applicable, $T_{w}$ is the duration of the window used by f-OFDM and $W_{\mathrm{GB}}$ is the width of the guard band used by FMT. We set $W_{\mathrm{GB}}$ so that FMT has the same spectral efficiency as OFDM. Finally, note that the
GFDM system we consider uses blocks of 5 symbols, which is a commonly used value [35].

Due to filtering, leakage interference for FBMC/OQAM, FBMC-PAM and f-OFDM is concentrated in the subcarrier directly adjacent to the active resource block. Hence, to take full advantage of the improved spectral properties of these waveforms, we only use 11 subcarriers per resource block instead of 12, leaving a guard band of 1 subcarrier between resource blocks. All other waveforms use the full 12 subcarriers since their sidelobes are larger and leakage interference spans multiple subcarriers. In these cases, adding a single guard subcarrier between resource blocks would offer little advantage and would just reduce the bandwidth efficiency of the waveforms, thereby reducing the rate achievable with them. Note that FMT is a special case in that it does not require a guard subcarrier between resource blocks due to its implementation, which places a guard band between every subcarrier.

\section{Interference Model}

To model interference between users using each of the aforementioned waveforms considered in this article, we follow the same approach as in [9], [10]. However, whereas our previous analyses were based on interference tables with a spectral granularity of one subcarrier spacing, we base the present system-level analysis on interference tables that are defined at the RB level. This is necessary to be able to carry out system level studies, as resource allocation and other procedures of the upper layers operate with a minimum granularity of one RB. Besides, whereas most studies on asynchronous networks rely on average values of interference [11], [15], we compute the interference between given pairs of waveforms for specific values of timing offset $\delta_{\mathrm{t}}$. Moreover, we also take CFO into account. We therefore generate three-dimensional interference tables which give the interference value at a given RB distance for each possible value of the timing offset, $\delta_{t}$, and $\mathrm{CFO}, \delta_{\mathrm{f}}$. We consider that the timing offset and CFO between users are uniformly distributed in a given interval so that $\delta_{\mathrm{t}} \sim U_{\left[-\delta_{\mathrm{t}}^{\max }, \delta_{\mathrm{t}}^{\max }\right]}$ and $\delta_{\mathrm{f}} \sim U_{\left[-\delta_{\mathrm{f}}^{\max }, \delta_{\mathrm{f}}^{\max }\right]}$. 

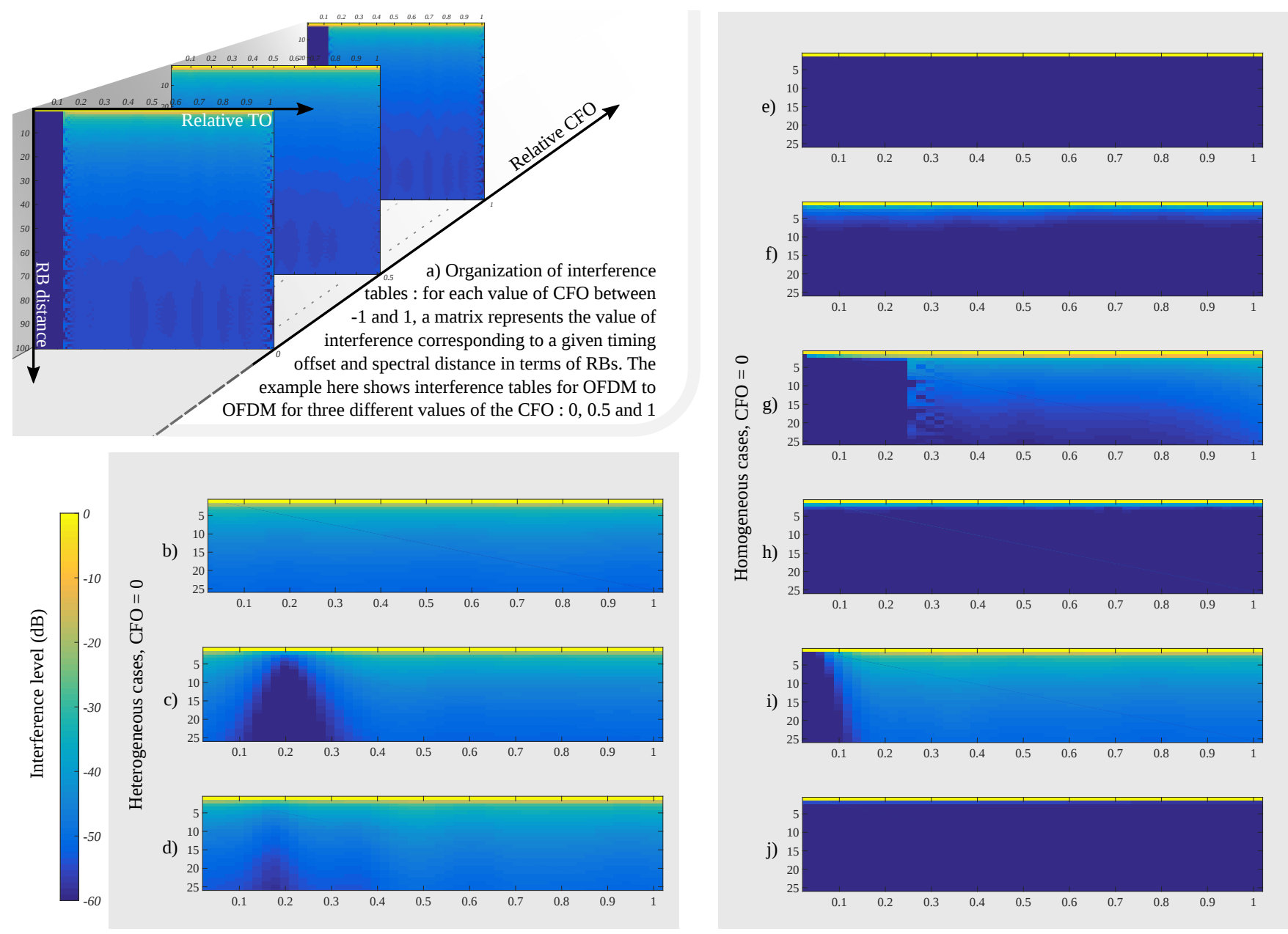

Fig. 2. Representative examples of interference tables. Timing offset is expressed in proportion of $T+T_{\mathrm{CP}}$ of a reference OFDM configuration and CFO is expressed relative to $\Delta \mathrm{F}$. Values lower than $-60 \mathrm{~dB}$ appear in dark blue.

a) Organization of interference tables and OFDM to OFDM example. b) FBMC/OQAM to OFDM. c) f-OFDM to OFDM. d) UFMC to OFDM. e) FBMC/OQAM to FBMC/OQAM. f) FBMC-PAM to FBMC-PAM. g) GFDM to GFDM. h) FMT to FMT. i) UFMC to UFMC. j) f-OFDM to f-OFDM.

In order to present our interference model, we display in Fig. 2 some of the interference tables that we generated and made available in our system-level simulator. In particular, we present in Fig. 2-a the structure of the interference tables as they are represented in our system level simulator. For each value of $\delta_{\mathrm{t}}$ and $\delta_{\mathrm{f}}$, our tables provide the corresponding level of interference, up to a maximum spectral distance of 100 RBs. Note that we consider heterogeneous scenarios in which DUEs use an alternative waveform and CUEs employ OFDM, and more advanced homogeneous scenarios in which both CUEs and DUEs use an alternative waveform. To model the interference between different users in these different setups, we therefore need to generate homogeneous interference tables, from a given waveform to the same waveform, and heterogeneous ones, from a given waveform to OFDM and from OFDM to a given waveform.

In Fig. 2, for each table Waveform $A$ to Waveform $B$, an interfering user using Waveform $A$ is active on an RB of index 0 , and we show the interference power seen by a victim user using Waveform $B$ at a given spectral distance specified in number of RBs, and for given values of the timing offset $\delta_{\mathrm{t}}$.
Note that, due to space limitations, we present interference tables only in the case where there is no CFO, i.e. $\delta_{\mathrm{f}}=0$, and only for spectral distances lower than $25 \mathrm{RBs}$.

Fig. 2-b shows the interference table from FBMC/OQAM to OFDM. Consistent with [41], we show that the interference to the OFDM receiver decreases very slowly in frequency and does not exhibit any particular behaviour related to the timing offset. This twofold observation holds true for interference from any FBMC waveform to an OFDM receiver or from an OFDM transmitter to any FBMC receiver. However, for waveforms that are filtered per block of subcarriers, such as filtered-OFDM and UFMC, the interference experienced by an OFDM receiver achieves a minimum point for specific values of the timing offset, as can be seen in Fig. 2-c and Fig. 2-d, respectively. This is due to the similarity of these waveforms to the OFDM scheme. Note that this observation is consistent with the analysis of Ahmed et al. in [42].

More diverse and interesting behaviours can be observed in homogeneous cases; as is commonly known, coexisting OFDM systems will not interfere with each other provided that they are synchronized within the CP duration, which is 


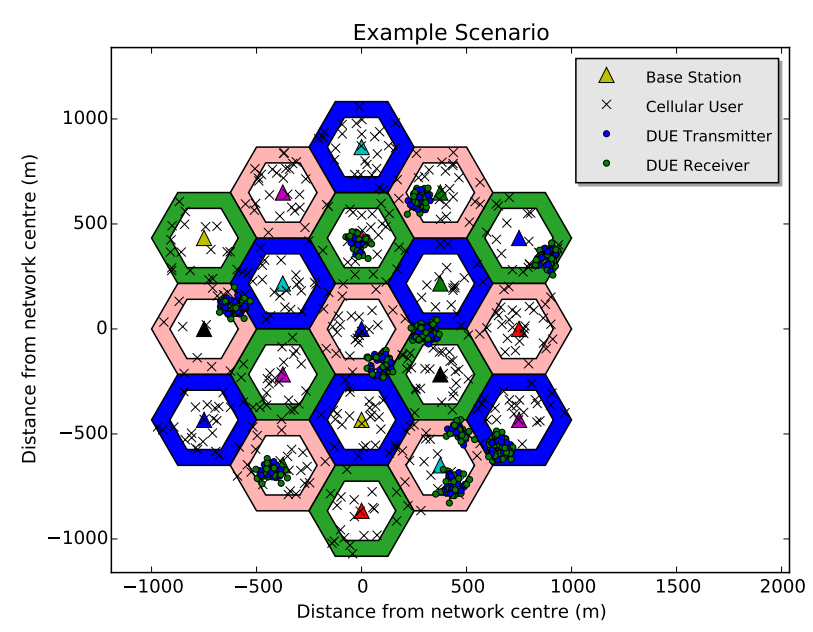

Fig. 3. Example scenario consisting of 19 cells, with each region coloured according to the spectral resources permitted for use. Each $x$ represents an ordinary cellular user, whereas DUEs involved in direct communication are clustered in groups.

verified in Fig. 2-a for $\delta_{\mathrm{f}}=0$. This statement also applies almost directly to GFDM systems, which achieve quasiorthogonality even if the timing offset is contained within the $\mathrm{CP}$ duration, as can be seen in Fig. 2-g. For both OFDM and GFDM systems, interference between users dramatically increases if their relative asynchronism goes beyond the cyclic prefix. On the other hand, FBMC waveforms that are based on linear convolution (Fig. 2-e,f,h) achieve good coexistence capabilities, with the injected interference dropping rapidly along the frequency axis irrespective of the timing offset value. As we can see in Fig. 2-e, the best performance is achieved by the FBMC/OQAM waveform, as no interference leaks onto adjacent resource blocks.

Filtered-OFDM (see Fig. 2-j) exhibits similar behaviour owing to its filtering at both the transmitter and the receiver. In contrast, UFMC (Fig. 2-h) only achieves good containment for timing offset values that are lower than the duration of the filter used. Outside of this interval, interference rapidly increases, which is consistent with what has been observed in [8]. This is because the particular implementation of UFMC considered here does not involve any windowing at the receiver. We highlight that the performance of certain waveforms, in particular GFDM and UFMC, could be improved by the use of additive windowing techniques at the transmitter and/or the receiver side. However, we intentionally do not implement these techniques in order to keep the focus on the intrinsic filtering properties offered by the waveforms under study. This is important as every additional filtering operation increases the overall complexity of the system.

\section{Evaluation of System Performance}

\section{A. Scenario Under Investigation}

We first present detailed results for the scenario defined by the parameters listed in Table II. For each set of results, we compare the asynchronous performance of all waveforms under consideration, and use the performance of synchronous OFDM as an baseline for comparison.

We highlight that the synchronous OFDM case serves as an idealistic baseline for comparison with the asynchronous cases and that, in reality, achieving synchronous communication for the DUEs would be challenging. This is true even if the D2D communication is network assisted [7]. The base station applies a timing advance to ensure all signals reach the base station simultaneously; however, due to the varying distances between DUE pairs, signals will not arrive at DUE receivers simultaneously and hence the DUE pairs will not be fully synchronised with one another. In addition, a D2D pair may span multiple cells, further complicating the issue.

We therefore assume quasi-orthogonality in which all timing offsets are absorbed by an extended CP of $20 \%^{2}$ for the synchronous OFDM baseline case. We also do not consider $\mathrm{CFO}$ in this case for two reasons. First, the scenario that we are considering typically consists of low mobility, resulting in negligible Doppler shifts and frequency offsets. Secondly, the 3GPP standards specify stringent frequency errors for UEs of less than +/- 0.1 parts per million (ppm) [43] compared to the carrier frequency received from the BS.

In contrast, for asynchronous scenarios, we consider timing offsets uniformly distributed in the range of 0 to $T+T_{C P}$, where $T$ is the length of an OFDM symbol and $T_{C P}$ is the length of the cyclic prefix. We also consider less stringent hardware-related frequency error requirements, with local oscillator (LO) inaccuracies of $+/-2.5 \mathrm{ppm}^{3}$ permitted.

The cell radius value of $250 \mathrm{~m}$ is based on the $3 \mathrm{GPP}$ LTE system scenarios [44], representing an urban macro-cell environment. The antenna gain values, noise figures, and the carrier frequency value are also based on [44]. The values for the maximum CUE transmit power, subcarrier spacing, and number of resource blocks are based on the LTE standard, with 50 resource blocks corresponding to a bandwidth of $10 \mathrm{MHz}$. The maximum DUE transmit power of $-5 \mathrm{dBm}$ was chosen as we found through experimentation that it yielded good results. The effects of varying the maximum DUE transmit power will be discussed later in this section.

Fig. 3 shows an example of a typical simulation scenario. We explore the case whereby each macro-cell is fully loaded, with all available resource blocks being utilised, and hence consider a large number of CUEs per square kilometre to ensure this. The parameters relating to the size and frequency of occurrence of clusters are scenario dependant. A cluster of radius $60 \mathrm{~m}$, containing 30 inter-communicating devices and with an average of 3 clusters per square kilometre might, for example, represent a factory in an urban area with moderate industrial activities.

Simulating the network for every possible combination of waveform pairs would be impractical and unnecessary. Hence,

\footnotetext{
${ }^{2}$ The value of $20 \%$ was chosen as it is similar to the size of the extended CP option in LTE.

${ }^{3}$ Generally, strict frequency error requirements require more accurate and expensive clocks. $2.5 \mathrm{ppm}$ is the stated frequency accuracy of the NI USRP292x range of devices.

${ }^{4}$ Noise power per $\mathrm{RB}$ is calculated using the expression $-174 \mathrm{dBm} / \mathrm{Hz}+10 \log _{10}(180 \mathrm{kHz})$, where $-174 \mathrm{dBm} / \mathrm{Hz}$ is the thermal noise and $180 \mathrm{kHz}$ is the bandwidth of an LTE RB.
} 
TABLE II

SIMULATION PARAMETERS

\begin{tabular}{|c|c|}
\hline Parameter & Value \\
\hline Cell Radius & $250 \mathrm{~m}$ \\
\hline Inner Radius & $163 \mathrm{~m}$ \\
\hline Number of Cells & 19 \\
\hline CUEs Per Square Km & 200 \\
\hline DUEs Per Cluster & 30 \\
\hline Clusters Per Square Km & 3 \\
\hline Average Cluster Radius & $60 \mathrm{~m}$ \\
\hline Average Tx-Rx Distance & $\begin{array}{l}\text { Uniformly distributed } \\
\text { in the range }[10,50] \mathrm{m}\end{array}$ \\
\hline Carrier Frequency & $2 \mathrm{GHz}$ \\
\hline Subcarrier Spacing $(\Delta f)$ & $15 \mathrm{kHz}$ \\
\hline Noise Per RB ${ }^{4}\left(\sigma_{\nu}^{2}\right)$ & $-116 \mathrm{dBm}$ \\
\hline Number RBs in system & 50 \\
\hline$P_{\mathrm{O} \_\mathrm{PUSCH}}$ & $-96 \mathrm{dBm}$ \\
\hline Max Tx Power CUE & $24 \mathrm{dBm}$ \\
\hline Max Tx Power DUE & $-5 \mathrm{dBm}$ \\
\hline BS Antenna Gain & $15 \mathrm{dBi}$ \\
\hline UE Antenna Gain & $0 \mathrm{dBi}$ \\
\hline BS Noise Figure & $5 \mathrm{~dB}$ \\
\hline UE Noise Figure & $9 \mathrm{~dB}$ \\
\hline Max Timing Offset & $T+T_{C P}$ \\
\hline $\begin{array}{l}\text { Max Local Oscillator (LO) } \\
\text { Inaccuracy }\end{array}$ & $2.5 \mathrm{ppm}$ \\
\hline Waveforms & $\begin{array}{c}\text { OFDM, FMT, } \\
\text { FBMC/OQAM, FBMC-PAM, } \\
\text { GFDM, f-OFDM, UFMC }\end{array}$ \\
\hline Number of Iterations & 10000 \\
\hline
\end{tabular}

we only examine the most realistic combinations:

1) Case 1: DUE pairs use an alternative waveform and CUEs continue to use OFDM.

2) Case 2: Both DUE pairs and CUEs use an alternative waveform.

We also examine the effects of the timing offset on the relative performance of all waveforms, ranging from perfectly synchronised to fully asynchronous communication. An analogous investigation is performed for CFO by varying local oscillator (LO) inaccuracies.

\section{B. System Performance}

1) DUE SINR Performance: Fig. 4 presents box plots summarizing the SINR distribution for DUEs according to each considered waveform couple. A solid horizontal line in each box represents the median, while the mean is marked with a dashed horizontal line. The ideal baseline OFDM case, assuming no timing or frequency offsets, performs quite well and achieves an average SINR value of approximately $22 \mathrm{~dB}$. This, however, reduces to approximately $13 \mathrm{~dB}$ when asynchronous communication is considered, with UFMC and GFDM exhibiting similar average values. This reduction in performance can be attributed to increased leakage interference between DUEs owing to the large sidelobes exhibited by these waveforms. When both CUEs and DUEs employ an alternative

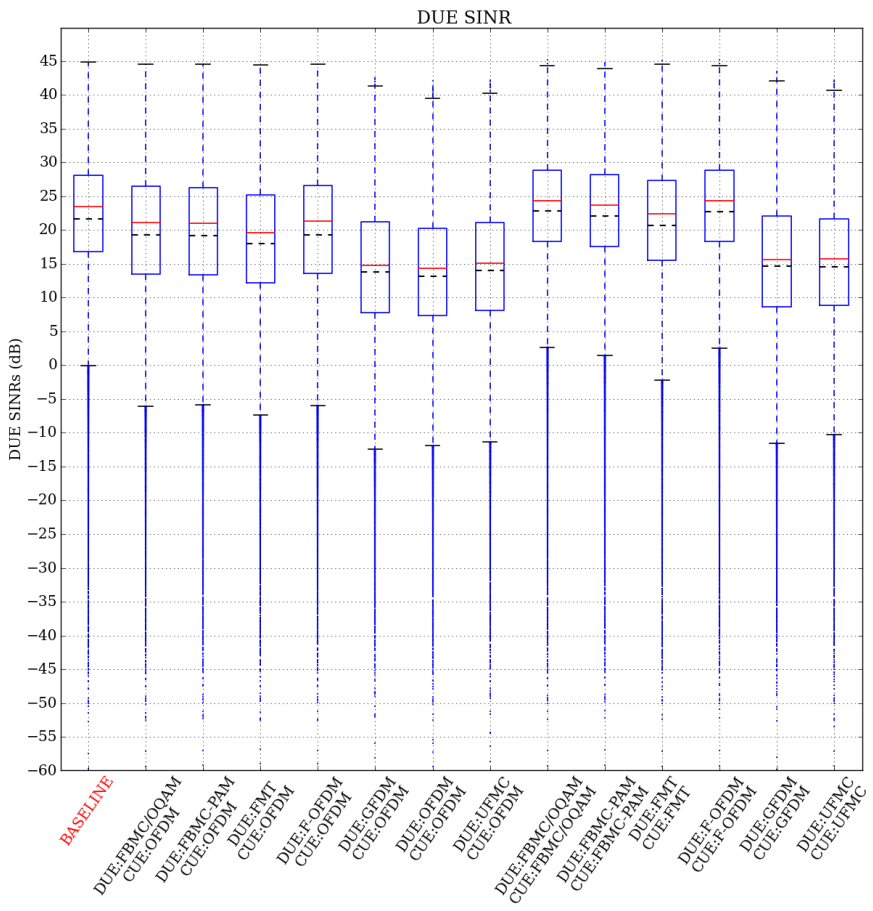

Fig. 4. The box plots of DUE SINR show that a large performance increase can be obtained by choosing an appropriate alternative waveform.

waveform in the set FBMC/OQAM, FMT, FBMC-PAM, $\mathrm{f}-\mathrm{OFDM}\}$, performance comparable to the baseline case is achieved even though communication is asynchronous, as the filtering operations substantially reduce the sidelobes of these waveforms. In addition, any leakage remaining after filtering is absorbed by the guard subcarrier for FBMC/OQAM, FMT, and f-OFDM, which explains how two waveforms with different filters, such as f-OFDM and FBMC/OQAM, can present with similar SINR values.

Interestingly, this same set of waveforms performs quite well in the coexistence scenarios in which CUEs use OFDM and DUEs use an alternative waveform, with average values approximately $3 \mathrm{~dB}$ less than in the baseline case, but up to $6 \mathrm{~dB}$ greater than asynchronous OFDM. Again, we can explain this by highlighting the increased spectral containment of these waveforms over OFDM, resulting in less inter-DUE interference.

We note that the number of outliers is relatively small (approx. 2.2\%) compared to the number of DUEs in the data set. The presence of outliers is not unusual; while the majority of DUEs will experience a similar SINR to one another, especially favourable or unfavourable channel conditions will inevitably result in DUEs with SINRs that are considerably higher or lower than average.

2) DUE Rate Performance: Fig. 5 shows the achieved rate of DUE pairs for each waveform. The greatest performance is achieved when both CUEs and DUEs use FBMC/OQAM, closely followed by FBMC-PAM. This is understandable, as these two waveforms have the best bandwidth efficiency (see Table I) out of alternative waveforms considered due to the fact that they do not employ a cyclic prefix. This 


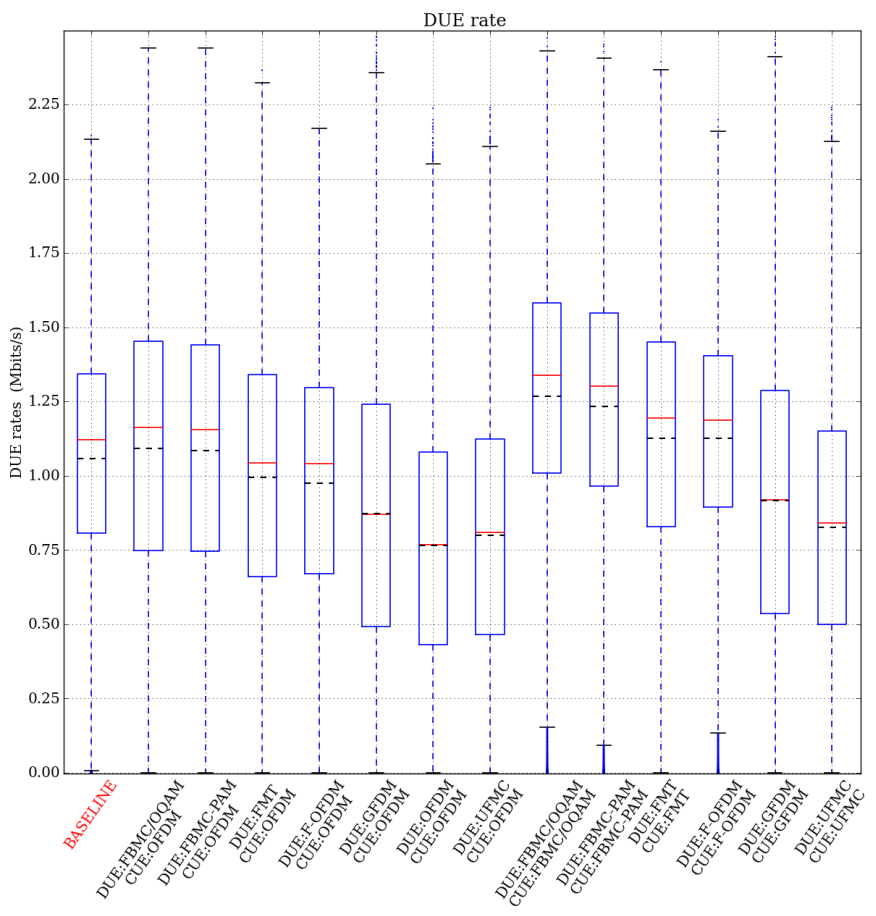

Fig. 5. Rate performance of DUEs taking into account bandwidth efficiency.

explains why FBMC/OQAM outperforms f-OFDM in terms of rate performance, despite both exhibiting similar SINR distributions in Fig. 4. Furthermore, recall from Section IV that FMT employs a guard band between each subcarrier, with the guard band width set so that FMT has the same spectral efficiency as OFDM.

In the coexistence scenarios, in which CUEs use OFDM and DUEs use an alternative waveform, both FBMC/OQAM and FBMC-PAM again exhibit the best performance. In both of these cases, the achieved rate is marginally greater than for the synchronous OFDM baseline case and approximately $43 \%$ greater than for the asynchronous OFDM case. This is an encouraging result, as machine-type DUEs could enjoy the benefits of asynchronous communication without suffering any degradation in performance. It also allows for the possibility that $5 \mathrm{G}$ will permit multiple waveforms, whereby different services employ the waveform that is best suited to them. We note that although the average data rate for these two cases is marginally greater than for the synchronous OFDM baseline case, it can be observed in Fig. 4 that the baseline case actually achieves a higher SINR. This can again be attributed to the bandwidth efficiencies of the waveforms, with the $20 \%$ efficiency loss due to the extended CP in the baseline case causing significant data rate degradation.

3) CUE Performance: It is imperative that CUE performance not be significantly degraded by the inclusion of MTC in the network. Fig. 6 demonstrates that the average DUE to CUE interference is quite low. This can be attributed to two factors: the low transmit power of DUEs $(-5 \mathrm{~dB})$ and the use of strict FFR.

In the synchronous baseline case, CUEs will still suffer slightly from leakage interference from DUEs, owing to the

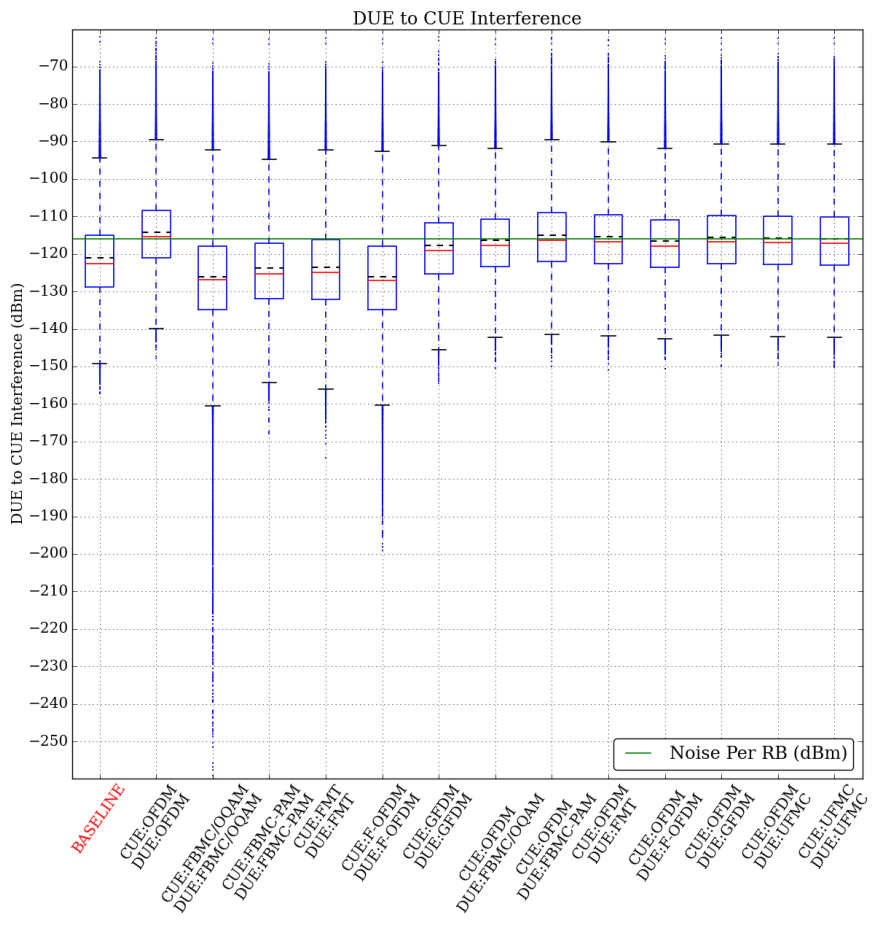

Fig. 6. The DUE to CUE interference is similar to the value of noise per resource block for coexistence cases.

fact that different cells in LTE are misaligned in time. The use of an appropriate alternative waveform by both sets of users can therefore assist in reducing the interference that CUEs experience from DUEs. Hence, in cases where both CUEs and DUEs use an alternative waveform from the set \{FBMC/OQAM, FMT, FBMC-PAM, f-OFDM $\}$, the interference experienced by CUEs is lower than in the baseline case.

In all other cases, the interference is comparable to the noise per RB. However, Fig. 6 demonstrates that employing a different waveform for DUEs, while CUEs continue to use OFDM, does little to mitigate DUE to CUE interference; its main benefit is to increase the performance of the DUEs themselves. This is because for CUEs, the interference from DUEs using the same RB will generally be a greater factor than leakage. In contrast, for DUEs, leakage interference from other DUEs in close proximity is the dominant type of interference, and hence they can benefit from adopting a waveform with better spectral localisation than OFDM.

The presence of outliers suggests that while the majority of CUEs suffer little degradation to their performance, a small number of users suffer a large reduction in performance. These users are victims of the specific spatial distribution of transmitting users at that instant, in which strict FFR and the low transmit power of DUEs fail to offer sufficient protection. In these cases, additional protection is needed to ensure that interference to CUEs is kept at an acceptable level and that the minority of users who suffer significant degradation can also obtain adequate performance. This may take the form of intelligent resource allocation schemes that aim to protect vulnerable CUEs by assigning resources in a manner that reduces DUE to CUE interference. As stated previously, such 
schemes are not within the scope of this paper as we are solely interested in demonstrating the effect on performance of employing different waveforms.

\section{DUE Transmit Power}

We investigate the effect that DUE transmit power has on system performance by varying the DUE transmit power from $-15 \mathrm{dBm}$ to $15 \mathrm{dBm}$ in $5 \mathrm{dBm}$ increments, while holding all other parameters at the same value as in Table II. We do not include cases in which both CUEs and DUEs use an alternative waveform, as we are more interested in the coexistence cases.

As intuition suggests, Fig. 7 shows that increasing the DUE transmit power will increase DUE SINR at the cost of increased interference to CUEs. The case in which DUEs employ OFDM for asynchronous communication exhibits the worst performance, as the large sidelobes of OFDM cause interference with neighbouring users in the resource grid. Leakage interference from D2D pairs in other cells will be present even in the synchronous case as neighbouring cells do not achieve time alignment. While FBMC/OQAM and fOFDM successfully mitigate this type of leakage interference, it becomes significant at high transmit powers for synchronous OFDM and, hence, the curve representing the baseline case begins to taper as the transmit power is increased. So, while the synchronous baseline case achieves the greatest performance for low transmit powers, it is overtaken by both FBMC/OQAM and f-OFDM at a transmit power of $7.5 \mathrm{dBm}$ as leakage from other cells becomes significant.

In particular, we draw the readers' attention to two points.

1) First, for the case in which DUEs do not use an alternative waveform from the set FBMC/OQAM, FMT, FBMCPAM, f-OFDM $\}$, successively higher transmit powers provide increasingly diminishing returns since increasing DUE transmit power will also increase the inter-DUE leakage interference. This is evident in Fig. 7, in which the set of curves at the bottom of the upper sub-plot gradually begin to level off as the DUE transmit power is increased.

2) Secondly, the benefit to DUEs of using an alternative waveform will be greater at higher values of DUE transmit power since inter-DUE leakage will be more prominent. However, as the DUE transmit power is increased, there is a linear increase in the interference experienced by CUEs.

The main consequence of these observations is that higher DUE transmit powers provide increasingly diminishing returns unless an alternative waveform that adequately mitigates leakage interference is employed. A maximum permissible transmit power should be chosen for DUEs that achieves a balance between adequate average DUE SINR and an acceptable level of interference to CUEs. The value of $-5 \mathrm{dBm}$ chosen in Table II reasonably achieves this, with DUEs achieving a SINR close to $20 \mathrm{~dB}$ when they employ FBMC/OQAM while limiting interference to CUEs to approximately the noise value per RB. We also note that we can trade off some DUE performance for reduced interference to CUEs. We observe, however, that the DUE to CUE interference is at a minimum for the synchronous
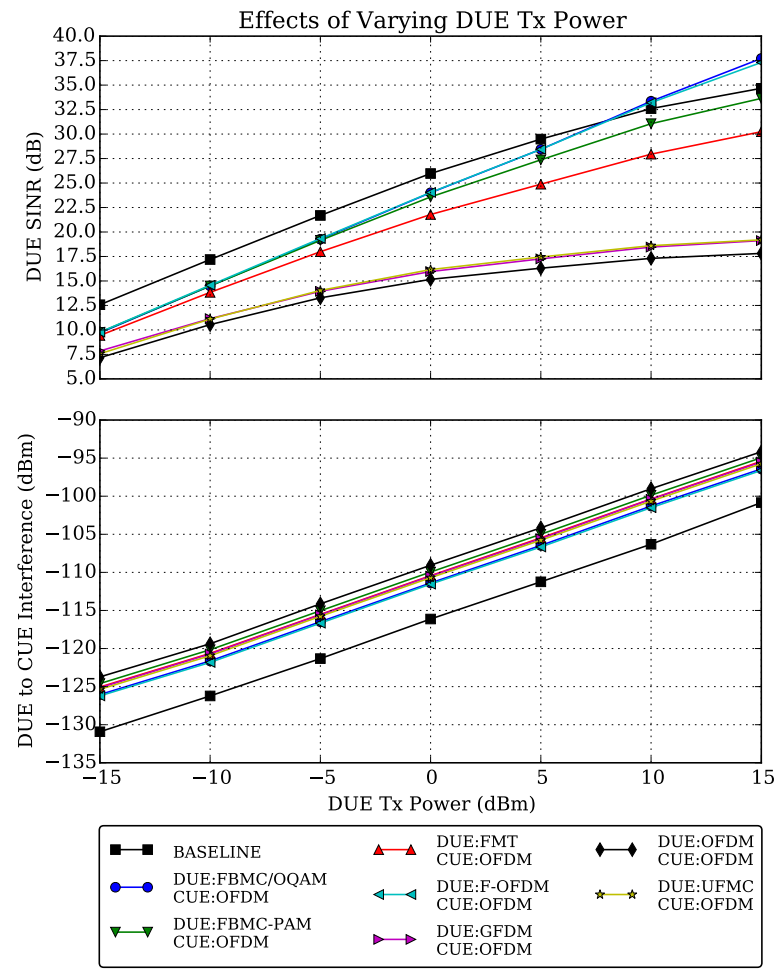

Fig. 7. Increasing DUE transmit power results in an increase in DUE SINR at the cost of increased interference to CUEs.

baseline case, as no leakage interference within the same cell is present.

\section{Cell Radius}

In this subsection, we investigate the influence that cell size has on performance by varying the cell radius from $200 \mathrm{~m}$ to $1000 \mathrm{~m}$ in $100 \mathrm{~m}$ increments while holding all other parameters at the same value as in Table II. We display the results in Fig. 8. For cell radii under $500 \mathrm{~m}$, we consider an urban environment and use the appropriate pathloss models for this scenario, while for cell radii greater than $500 \mathrm{~m}$, we consider a suburban environment.

At the smallest cell radius considered $(200 \mathrm{~m})$, average DUE SINR is at its lowest and average DUE to CUE interference is at its greatest. This is understandable, and readily explained as follows. According to the strict FFR scheme employed, DUEs reuse the resources of CUEs in neighbouring reuse regions. At small cell sizes, the average distance between devices in neighbouring reuse regions is reduced. This results in greater CUE to DUE interference and reduces DUE SINR. As the cell radius increases, so too does the distance between reuse regions, and DUE SINR increases. This increase is mainly observed at smaller cell sizes; at large cell sizes, CUE to DUE interference is almost negligible and further increases to cell radius result in little or no increase in DUE SINR.

DUE to CUE interference, on the other hand, occurs at base stations. In small cells, the average distance between clusters and the base stations serving neighbouring reuse regions is shorter, resulting in higher DUE to CUE interference. This is 

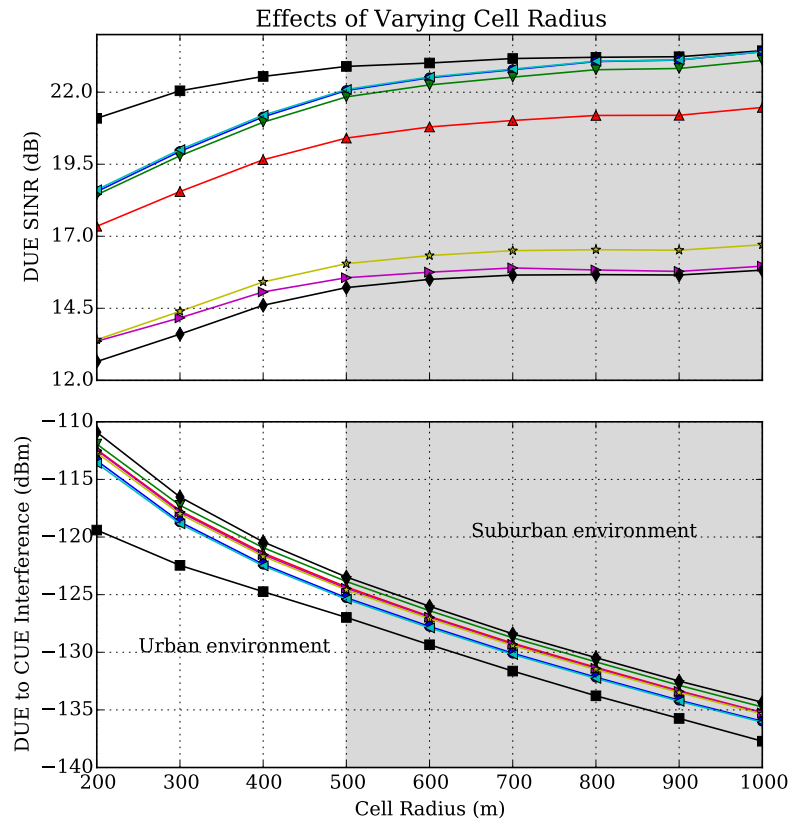

\begin{tabular}{|c|c|c|c|c|c|}
\hline 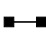 & BASELINE & 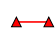 & $\begin{array}{l}\text { DUE:FMT } \\
\text { CUE:OFDM }\end{array}$ & $\longleftrightarrow$ & $\begin{array}{l}\text { DUE:OFDM } \\
\text { CUE:OFDM }\end{array}$ \\
\hline$\bullet$ & $\begin{array}{l}\text { DUE:FBMC/OQAM } \\
\text { CUE:OFDM }\end{array}$ & $\triangleleft \triangleleft$ & $\begin{array}{l}\text { DUE:F-OFDM } \\
\text { CUE:OFDM }\end{array}$ & $\not \vec{~}$ & $\begin{array}{l}\text { DUE:UFMC } \\
\text { CUE:OFDM }\end{array}$ \\
\hline$\nabla \longrightarrow$ & $\begin{array}{l}\text { DUE:FBMC-PAM } \\
\text { CUE:OFDM }\end{array}$ & $\triangleright$ & $\begin{array}{l}\text { DUE:GFDM } \\
\text { CUE:OFDM }\end{array}$ & & \\
\hline
\end{tabular}

Fig. 8. As the cell radius increases, DUE SINR increases and reduction in CUE SINR decreases.

evidenced in the lower sub-plot in Fig. 8, in which we observe that the average DUE to CUE interference decreases as the cell radius increases. Therefore, as the cell size increases, average DUE to CUE interference decreases and average DUE SINR increases. Essentially, the greater the cell size the more protection strict FFR offers against the various types of interference, as the reuse regions are further apart.

Over the range of cell radii considered, synchronous OFDM provides the best performance and asynchronous OFDM provides the worst. However, as the cell radius increases, the interference from CUEs in neighbouring reuse regions to DUEs is reduced and the performance of several alternative waveforms approaches that of synchronous OFDM. At large cell sizes, even further gains are achievable as DUEs could transmit at a higher power without affecting CUEs.

\section{E. Cluster Radius}

We investigate the impact that cluster radius has on performance. We present the results in Fig. 9, varying the cluster radius from $30 \mathrm{~m}$ to $100 \mathrm{~m}$ in $10 \mathrm{~m}$ increments. Reducing the cluster radius necessitates a corresponding change in the distance between a DUE transmitter and receiver, which we modelled using a uniform random variable. Accordingly, we choose the parameters $a$ and $b$, representing the minimum and maximum Tx-Rx distances, respectively, of the uniform random variable $U_{[a, b]}$ as follows: $a=5 \mathrm{~m} ; b=$ (cluster radius) $-10 \mathrm{~m}$.

Increasing the cluster radius has two opposing influences on DUE SINR. On the one hand, it results in reduced interDUE interference, which should boost the SINR. On the

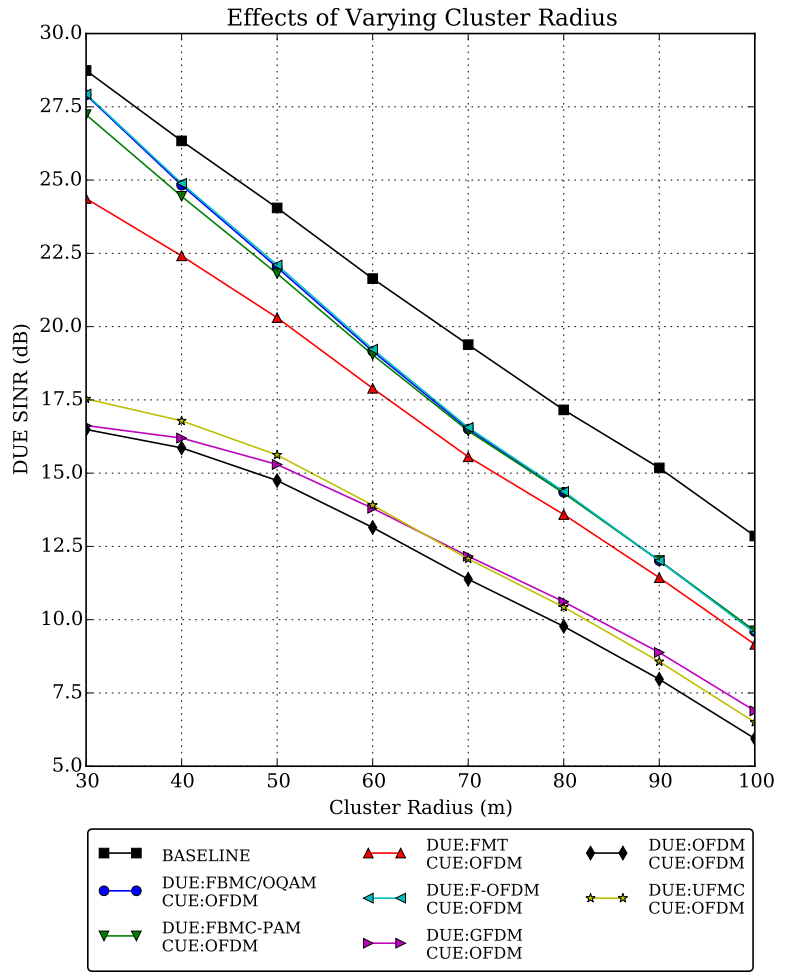

Fig. 9. Employing an appropriate alternative waveform for DUEs yields the greatest benefit in small clusters in which inter-DUE leakage interference is most significant.

other hand, it also results in reduced received signal power, which should cause the SINR to decrease. In Fig. 9, we see that the reduction in received power is more influential and DUE SINR decreases as cluster radius increases. We concede, however, that this is somewhat dependant on how the distance between DUE transmitters and receivers is modelled (such as the parameters $a$ and $b$ ), as this affects by how much the received power will decrease. We also note that for small cluster sizes, and in cases where DUEs do not use a waveform in the set $\{$ FBMC/OQAM, FMT, FBMC-PAM, f-OFDM , SINR decreases slowly at first as the reduction in inter-DUE interference is almost significant enough to counter-act the effect of lower received signal powers.

Reducing the cluster radius increases the density of DUEs in the cluster, resulting in greater inter-DUE interference. Hence, employing an appropriate alternative waveform for DUEs yields the greatest benefit in dense clusters in which inter-DUE leakage interference is most significant. The synchronous baseline case again performs the best; however, the performance for cases where DUEs use a waveform in the set $\{$ FBMC/OQAM, FBMC-PAM, f-OFDM $\}$ approach that of the baseline for small cluster sizes. This can be attributed to reduced leakage interference from CUEs in the same reuse region, as smaller clusters are less likely to encompass CUEs in the same cell. 


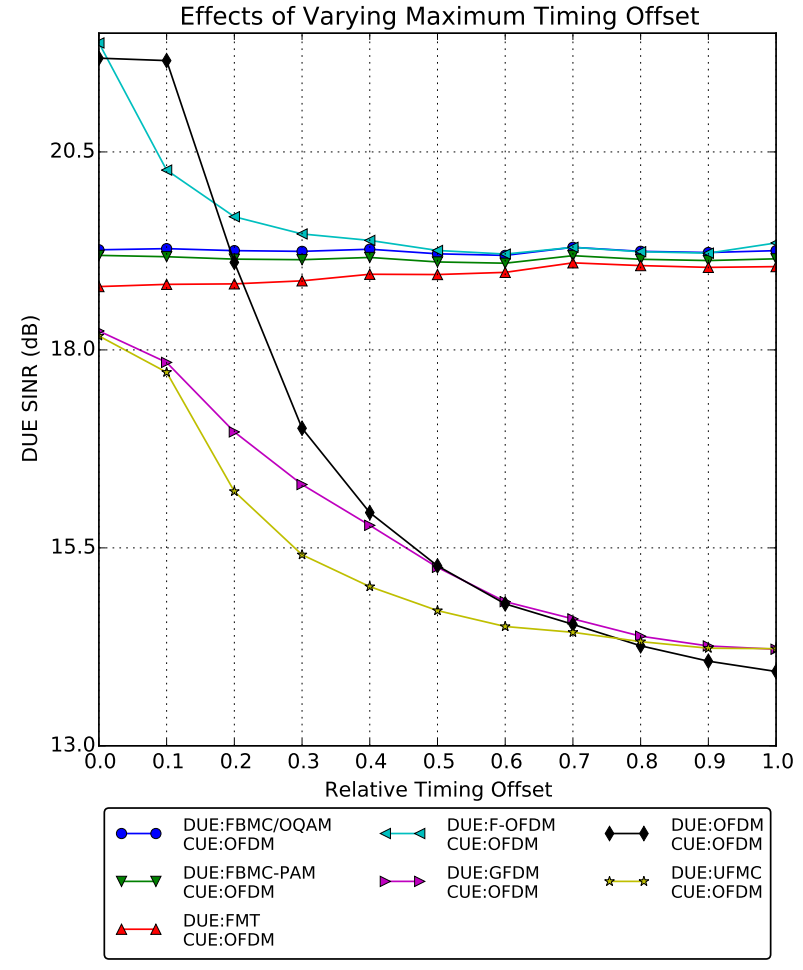

Fig. 10. DUE SINR performance as the maximum permitted timing offset is varied.

\section{F. Amount of Time and Frequency Misalignment between Devices}

The final parameters whose influence on performance we investigate are the maximum permitted timing offset and CFO. Both CFO and TO affect DUE performance similarly, and so it makes sense to isolate them when studying their effects on performance. Hence, when examining the effect of timing offset on DUE performance, we consider a case involving no CFO. Conversely, when investigating the effects of CFO, we consider devices to be perfectly aligned in time.

1) Maximum Possible Timing Offset: We vary the maximum permissible timing offset as a fraction of the time spacing between two OFDM symbols from 0 (full synchronism) to 1 (full asynchronism) in 0.1 increments. Limiting the maximum permissible timing offset corresponds to a case in which coarse alignment has been obtained; for example, 0.2 would correspond to the case in which devices are synchronised to within $20 \%$ of an OFDM symbol time.

Fig. 10 illustrates the results. The black line representing the case whereby both DUEs and CUEs use OFDM will be our baseline for comparison, and it can be seen that SINR drops rapidly when the timing offset is greater than the cyclic prefix, as the timing offsets are no longer fully absorbed by the cyclic prefix. The cyclic prefix duration $T_{\mathrm{CP}}$ for OFDM is $12.5 \%$ of the symbol duration $T$. We can divide the rest of the graph into two scenarios:

i) Scenario in which DUEs use a waveform in the set $\{G F D M, U F M C\}$, and CUEs use OFDM: These curves become quite similar as the maximum permissible timing offset increases, and are out-performed by our baseline OFDM-OFDM case. This seems surprising at first glance, but can be explained. Indeed, we saw in Fig. 2 that, with the chosen parameters, UFMC and GFDM still cause a significant amount of interference between coexisting users in homogeneous links in which both users are deploying one of these waveforms; thus, inter-DUE interference is quite important if DUEs use either GFDM or UFMC. Moreover, OFDM based users are orthogonal to one another as long as $\delta_{\mathrm{t}}$ is contained in the $\mathrm{CP}$ duration. However, GFDM or UFMC users never achieve orthogonality with OFDM users, which explains that if CUEs use OFDM, CUE to DUE interference is on average more significant if DUEs use UFMC or GFDM than if they also employ OFDM.

ii) Coexistence scenario in which DUEs use a waveform in the set $\{F B M C / O Q A M, F B M C-P A M, f-O F D M, F M T\}$ and CUEs use OFDM: As the timing offset increases, the curves exhibit similar performance. At a maximum timing offset, the benefit to using one of these alternative waveforms for DUEs is considerable, while for very low timing offsets $(<20 \%)$, they are outperformed by the baseline OFDM-OFDM case, since the CP in OFDM absorbs much of the timing offset. With the exception of f-OFDM, the performance of these waveforms varies little according to the timing offset, as these waveforms all exhibit excellent spectral localisation. In addition, FBMC/OQAM and FBMC-PAM both use a guard subcarrier while FMT is similarly protected by its inbuilt guards. F-OFDM has an interesting behaviour, as it is the only waveform that is affected differently by OFDM according to the value of $\delta_{\mathrm{t}}^{\max }$. This is due to the fact that for small timing offsets, f-OFDM and OFDM achieve quasi-orthogonality, which is then lost as $\delta_{\mathrm{t}}$ increases.

2) Maximum Possible CFO: Having investigated the effect of TO, we now examine the relative performance of the waveforms under various levels of CFO. The LO inaccuracy is varied from $0 \mathrm{ppm}$ to $3.5 \mathrm{ppm}$ in increments of 0.5 , corresponding to frequency offsets of $+/-0 \mathrm{kHz}$ to $+/-7 \mathrm{kHz}$ in $1 \mathrm{kHz}$ increments at a carrier frequency of $2 \mathrm{GHz}$.

In Fig. 11, for the case in which OFDM is used by both sets of users, we observe that the average DUE SINR reduces as the frequency offsets become greater. This can be attributed to OFDM's large sidelobes, resulting in significant interference leakage to and from other users. In a similar fashion to the study on the effects of TO, we again take the case in which both sets of users employ OFDM to be our baseline case, and divide the rest of Fig. 11 into two scenarios:

i) Scenario in which DUEs use a waveform in the set \{GFDM, UFMC $\}$, and CUEs use OFDM: When DUEs employ GFDM or UFMC, DUE SINR decreases as the maximum possible LO inaccuracy is increased; however, the decrease occurs at a lower rate than for OFDM since OFDM possess the largest sidelobes. For low LO inaccuracies, the baseline OFDM case outperforms the scenarios in which CUEs use OFDM and DUEs use either UFMC or GFDM. This is because OFDM users 


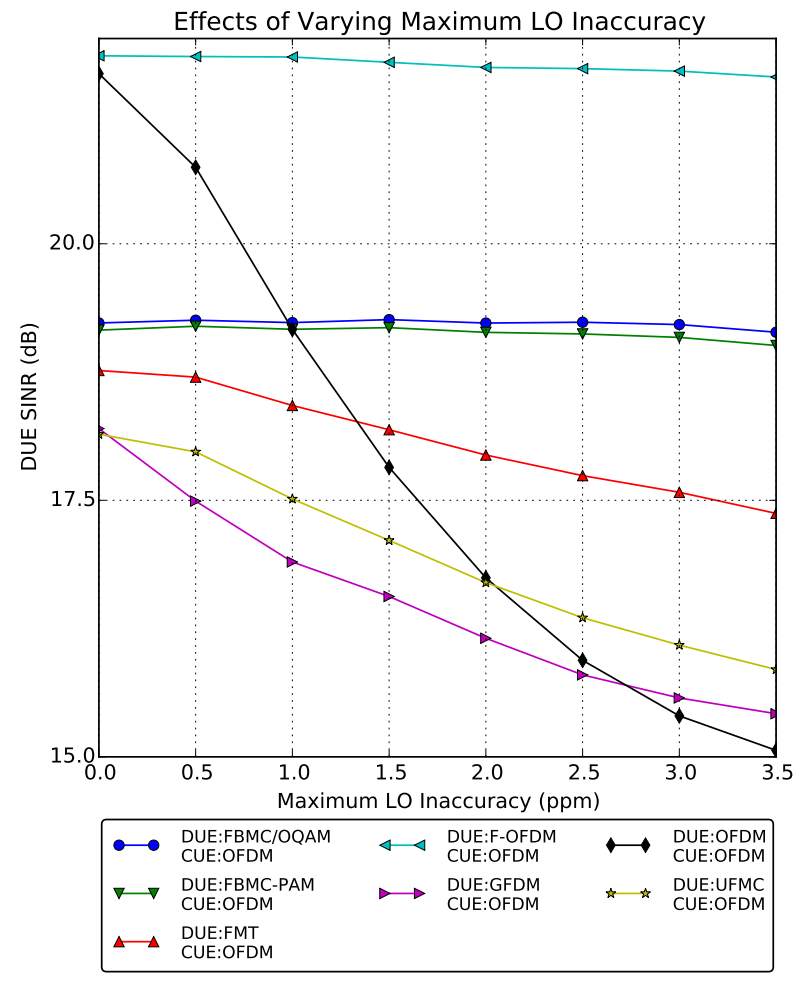

Fig. 11. DUE SINR performance as the maximum CFO is varied.

achieve near orthogonality at low CFOs, while GFDM or UFMC users never achieve orthogonality with OFDM users. However, as the LO inaccuracy is increased, OFDM suffers from increasingly large interference leakage owing to its sidelobes and the waveform choices involving UFMC or GFDM begin to outperform the baseline OFDM case.

ii) Coexistence scenario in which DUEs use a waveform in the set $\{F B M C / O Q A M, F B M C-P A M, f-O F D M, F M T\}$ and CUEs use OFDM: The waveform choices involving FBMC/OQAM, FBMC-PAM, and f-OFDM are largely unaffected by varying $\mathrm{CFO}$, as evidenced by the horizontal lines in Fig. 11. At the LO inaccuracies considered, frequency offsets are contained within $+/$ - half a subcarrier. Given that these schemes use a guard band of half a subcarrier at either side of an RB, and that leakage is confined within a similar range for these alternative waveforms, it is not surprising that very little variation in performance is observed as the CFO is increased. FMT, on the other hand, uses 12 subcarriers per RB. Hence, we observe that the SINR performance of DUEs using FMT reduces as the maximum possible LO inaccuracy is increased. The waveform choices involving FBMC-PAM and FBMC/OQAM only begin to outperform the baseline OFDM case after approximately $1 \mathrm{ppm}$. For DUE users using FMT, improvements in SINR over the baseline case are only observed after a maximum LO inaccuracy of $1.3 \mathrm{ppm}$ (based on an interpolated value). Similar to before, this is because OFDM achieves quasi-orthogonality at low CFO, but suffers significant degradation as CFO increases. Out of the waveform couples considered in this scenario, f-OFDM exhibits the best performance and is never outperformed by the baseline OFDM case. Similar to OFDM, f-OFDM achieves quasi-orthogonality at low CFO. However, dissimilar to OFDM, it is protected by its filtering and guard subcarrier as CFO increases and hence does not suffer the performance decrease experienced by OFDM.

\section{CONCLUding REMARKS}

The results presented in this paper were obtained through simulations, but are built upon theoretical analysis performed at the physical layer which characterises leakage interference between various waveform pairs. In fact, one of the main motivations for the paper is demonstrating how the wellresearched properties of waveforms translate to performance at a system-level in realistic 5G scenarios. In this pursuit, simulation is an ideal tool, as it permits us to achieve a high level of realism in our investigations.

When only the SINR metric is considered, the best results are obtained when either synchronous OFDM is used, or both sets of users employ a waveform from the set [FBMC/OQAM, FMT, FBMC-PAM, f-OFDM $\}$. When the achieved rate is instead considered, taking bandwidth efficiency into account, the case in which machine-type DUEs operate asynchronously and both sets of users employ FBMC/OQAM achieves the greatest performance.

As suggested in the previous section, the performance of a waveform in asynchronous communication depends on its sidelobes. Waveforms with very small sidelobes result in less inter-DUE leakage and hence perform the best. We note that the size of a waveform's sidelobes depends largely on the filtering applied, with many filter implementations existing. For example, the performance of FBMC/OQAM could be further improved by using an optimized filter such as the one suggested in [45]. However, as it was not possible to consider every possible filter, and for the sake of fair comparison, we chose filters and parameters that were representative of the most common implementations in the literature.

Promisingly, we also showed that good performance can be obtained when DUEs operate asynchronously and use a different waveform to CUEs, paving the way for the possibility of the coexistence of waveforms in 5G for different use cases, a paradigm shift from previous generations. In particular, when FBMC/OQAM is used by DUEs, the average achieved rate is marginally greater than the synchronous OFDM baseline case, and $43 \%$ greater than the asynchronous OFDM case. We also note that these figures are conservative, as they assume perfect synchronisation in the baseline case.

The results indicate that the biggest drawback to using asynchronous communication is the increased interference to cellular users. Unfortunately, employing a different waveform for DUEs does little to reduce this type of interference. We note, however, that interference can typically be kept low through the use of strict FFR and low DUE transmit powers.

To conclude, we have shown that it is feasible for cellular networks to serve clustered 5G MTC use-cases, such as 
smart factories, using asynchronous direct communication. In particular, we highlighted the benefits to DUEs of using an alternative waveform to reduce leakage interference, and suggested that $5 \mathrm{G}$ may permit the coexistence of waveforms. Hence, by employing a waveform with improved spectral localization compared to OFDM, such as FBMC/OQAM, DUEs can avail of the benefits of asynchronous communication without suffering a performance loss, even if regular CUEs continue to use OFDM.

\section{REFERENCES}

[1] C. Sexton, N. Kaminski, J. Marquez-Barja, N. Marchetti, and L. A. DaSilva, "5G: Adaptable Networks Enabled by Versatile Radio Access Technologies," IEEE Communications Surveys \& Tutorials, vol. 19, no. 2, pp. 688-720, Second Quarter 2017.

[2] Y. Cao, T. Jiang, M. He, and J. Zhang, "Device-to-Device Communications for Energy Management: A Smart Grid Case," IEEE Journal on Selected Areas in Communications, vol. 34, no. 1, pp. 190-201, Jan. 2016.

[3] M. Bagaa, A. Ksentini, T. Taleb, R. Jantti, A. Chelli, and I. Balasingham, "An efficient D2D-based strategies for machine type communications in 5G mobile systems," in 2016 IEEE Wireless Communications and Networking Conference, April 2016, pp. 1-6.

[4] J. Zhao, K. K. Chai, Y. Chen, J. Schormans, and J. Alonso-Zarate, "Joint mode selection and resource allocation for machine-type D2D links," Transactions on Emerging Telecommunications Technologies, vol. 28, no. 2, p. e3000, Nov. 2017.

[5] G. Steri, G. Baldini, I. N. Fovino, R. Neisse, and L. Goratti, "A novel multi-hop secure LTE-D2D communication protocol for IoT scenarios," in 2016 23rd International Conference on Telecommunications (ICT), May 2016, pp. 1-6.

[6] Y. Cao, T. Jiang, and Z. Han, "A Survey of Emerging M2M Systems: Context, Task, and Objective," IEEE Internet of Things Journal, vol. 3, no. 6, pp. 1246-1258, Dec. 2016.

[7] X. Lin, J. G. Andrews, A. Ghosh, and R. Ratasuk, "An overview of 3GPP device-to-device proximity services," IEEE Communications Magazine, vol. 52, no. 4, pp. 40-48, Apr. 2014.

[8] A. Aminjavaheri, A. Farhang, A. RezazadehReyhani, and B. FarhangBoroujeny, "Impact of timing and frequency offsets on multicarrier waveform candidates for 5G," in 2015 IEEE Signal Processing and Signal Processing Education Workshop (SP/SPE), Aug. 2015, pp. 178 183.

[9] C. Sexton, Q. Bodinier, A. Farhang, N. Marchetti, F. Bader, and L. A. DaSilva, "Coexistence of OFDM and FBMC for Underlay D2D Communication in 5G Networks," in Proc. of 2016 IEEE Global Communications Conference Workshops (Globecom Wkshps), Washington D.C., Dec. 2016, pp. 1-7.

[10] Q. Bodinier, A. Farhang, F. Bader, H. Ahmadi, J. Palicot, and L. A. DaSilva, "5G Waveforms for Overlay D2D Communications: Effects of Time-Frequency Misalignment," in Proc. of 2016 IEEE International Conference on Communications (ICC), Kuala Lumpur, May 2016.

[11] Y. Medjahdi, M. Terré, D. L. Ruyet, and D. Roviras, "Interference tables: a useful model for interference analysis in asynchronous multicarrier transmission," EURASIP Journal on Advances in Signal Processing, vol. 2014, no. 54, pp. 1-17, Apr. 2014

[12] Q. Bodinier, F. Bader, and J. Palicot, "On Spectral Coexistence of CPOFDM and FB-MC Waveforms in 5G Networks," IEEE Access, vol. 5, pp. 13883-13900, Jul. 2017.

[13] P. Wu, P. C. Cosman, and L. B. Milstein, "Resource Allocation for Multicarrier Device-to-Device Video Transmission: Symbol Error Rate Analysis and Algorithm Design," IEEE Transactions on Communications, vol. 65, no. 10, pp. 4446-4462, Oct. 2017.

[14] M. Pischella, R. Zakaria, and D. L. Ruyet, "Resource Block level power allocation in asynchronous multi-carrier D2D communications," IEEE Communications Letters, vol. 21, no. 4, pp. 813-816, Apr. 2017.

[15] — "Weighted sum rate maximization with filtered multi-carrier modulations for D2D underlay communications," in Proc. of 2016 IEEE 27th Annual International Symposium on Personal, Indoor, and Mobile Radio Communications (PIMRC), Valencia, Sep. 2016, pp. 1-6.

[16] Y. Li, X. Sha, and L. Ye, "Downlink Resource Sharing for D2D Communications in a Filtered OFDM System," in Proc. of 2016 IEEE 83rd Vehicular Technology Conference (VTC Spring), Sydney, May 2016, pp. 1-6.
[17] M. Mukherjee, L. Shu, Y. Zhang, Z. Zhou, and K. Wang, "Joint Power and Reduced Spectral Leakage-Based Resource Allocation for D2D Communications in 5G," in Proc. of the 15th International Conference on Algorithms and Architectures for Parallel Processing (ICA3PP), Zhangjiajie, Nov. 2015, pp. 244-258.

[18] H. Xing and M. Renfors, "Investigation of filter bank based deviceto-device communication integrated into OFDMA cellular system," in Proc. of the 11th International Symposium on Wireless Communications Systems (ISWCS), Barcelona, Aug. 2014, pp. 513-518.

[19] H. S. Chae, J. Gu, B. G. Choi, and M. Y. Chung, "Radio resource allocation scheme for device-to-device communication in cellular networks using fractional frequency reuse," in Proc. of The 17th Asia Pacific Conference on Communications (APCC), Kota Kinabalu, Oct. 2011, pp. $58-62$.

[20] Z. Zhang, R. Q. Hu, Y. Qian, and A. Papathanassiou, "D2D Communication Underlay in Uplink Cellular Networks with Fractional Power Control and Fractional Frequency Reuse," in Proc. of 2015 IEEE Global Communications Conference (Globecom), San Diego, Dec. 2015, pp. 17.

[21] S. Gupta, S. Kumar, R. Zhang, S. Kalyani, K. Giridhar, and L. Hanzo, "Resource Allocation for D2D Links in the FFR and SFR Aided Cellular Downlink," IEEE Transactions on Communications, vol. 64, no. 10, pp. 4434-4448, Oct. 2016.

[22] N. Saquib, E. Hossain, and D. Kim, "Fractional frequency reuse for interference management in LTE-advanced hetnets," IEEE Wireless Communications, vol. 20, no. 2, pp. 113-122, Apr. 2013.

[23] T. Novlan, J. G. Andrews, I. Sohn, R. K. Ganti, and A. Ghosh, "Comparison of Fractional Frequency Reuse Approaches in the OFDMA Cellular Downlink," in Proc. of 2010 IEEE Global Communications Conference (Globecom). Miami: IEEE, Dec. 2010, pp. 1-5.

[24] C. Cox, An introduction to LTE: LTE, LTE-advanced, SAE and $4 G$ mobile communications. John Wiley \& Sons, 2012, ch. 8.8.1, pp. $157-158$.

[25] K. Doppler, M. Rinne, C. Wijting, C. B. Ribeiro, and K. Hugl, "Deviceto-device communication as an underlay to LTE-advanced networks," IEEE Communications Magazine, vol. 47, no. 12, pp. 42-49, Dec. 2009.

[26] C.-H. Yu, K. Doppler, C. B. Ribeiro, and O. Tirkkonen, "Resource Sharing Optimization for Device-to-Device Communication Underlaying Cellular Networks," IEEE Transactions on Wireless Communications, vol. 10, no. 8, pp. 2752-2763, Aug. 2011.

[27] P. Kyösti, J. Meinilä, L. Hentilä, X. Zhao, T. Jämsä, C. Schneider, M. Narandzic, M. Milojevic, A. Hong, J. Ylitalo et al., "WINNER II channel models," WINNER II Public Deliverable, pp. 42-44, 2007.

[28] J. Abdoli, M. Jia, and J. Ma, "Filtered OFDM: A new waveform for future wireless systems," in Proc. of 2015 IEEE 16th International Workshop on Signal Processing Advances in Wireless Communications (SPAWC), Stockholm, Jun. 2015, pp. 66-70.

[29] D. Chen, X. G. Xia, T. Jiang, and X. Gao, "Properties and Power Spectral Densities of CP Based OQAM-OFDM Systems," IEEE Transactions on Signal Processing, vol. 63, no. 14, pp. 3561-3575, Jul. 2015.

[30] X. Gao, W. Wang, X. G. Xia, E. K. S. Au, and X. You, "Cyclic Prefixed OQAM-OFDM and its Application to Single-Carrier FDMA," IEEE Transactions on Communications, vol. 59, no. 5, pp. 1467-1480, May 2011.

[31] T. Wang, J. G. Proakis, and J. R. Zeidler, "Interference Analysis of Filtered Multitone Modulation Over Time-Varying Frequency- Selective Fading Channels," IEEE Transactions on Communications, vol. 55, no. 4, pp. 717-727, Apr. 2007.

[32] B. Farhang-Boroujeny, "OFDM Versus Filter Bank Multicarrier," IEEE Signal Processing Magazine, vol. 28, no. 3, pp. 92-112, May 2011.

[33] P. Siohan, C. Siclet, and N. Lacaille, "Analysis and design of OFDM/OQAM systems based on filterbank theory," IEEE Transactions on Signal Processing, vol. 50, no. 5, pp. 1170-1183, May 2002.

[34] M. Bellanger, D. Mattera, and M. Tanda, "A filter bank multicarrier scheme running at symbol rate for future wireless systems," in Proc. of 2015 Wireless Telecommunications Symposium (WTS), New York City, Apr. 2015, pp. 1-5.

[35] N. Michailow, M. Matth, I. S. Gaspar, A. N. Caldevilla, L. L. Mendes, A. Festag, and G. Fettweis, "Generalized frequency division multiplexing for 5th generation cellular networks," IEEE Transactions on Communications, vol. 62, no. 9, pp. 3045-3061, Sept 2014.

[36] H. Lin and P. Siohan, "Multi-carrier modulation analysis and WCPCOQAM proposal," EURASIP Journal on Advances in Signal Processing, vol. 2014, no. 1, p. 79, May 2014.

[37] B. Farhang-Boroujeny and H. Moradi, "OFDM Inspired Waveforms for 5G," IEEE Communications Surveys \& Tutorials, vol. 18, no. 4, pp. 2474-2492, Fourth Quarter 2016. 
[38] V. Vakilian, T. Wild, F. Schaich, S. ten Brink, and J.-F. Frigon, "Universal-filtered multi-carrier technique for wireless systems beyond LTE," in Proc. of 2013 IEEE Global Communications Conference Workshops (Globecom Wkshps), Atlanta, Dec. 2013, pp. 223-228.

[39] P. Banelli, S. Buzzi, G. Colavolpe, A. Modenini, F. Rusek, and A. Ugolini, "Modulation Formats and Waveforms for 5G Networks: Who Will Be the Heir of OFDM?: An overview of alternative modulation schemes for improved spectral efficiency," IEEE Signal Processing Magazine, vol. 31, no. 6, pp. 80-93, Nov. 2014.

[40] R. Gerzaguet, N. Bartzoudis, L. G. Baltar, V. Berg, J.-B. Doré, D. Kténas, O. Font-Bach, X. Mestre, M. Payaró, M. Färber, and K. Roth, "The 5G candidate waveform race: a comparison of complexity and performance," EURASIP Journal on Wireless Communications and Networks, vol. 2017, no. 1, p. 13, Jan. 2017.

[41] Q. Bodinier, F. Bader, and J. Palicot, "Coexistence in 5G: Analysis of Cross-Interference between OFDM/OQAM and Legacy Users," in Proc. of 2016 IEEE Global Communications Conference Workshops (Globecom Wkshps), Washington D.C., Dec. 2016.

[42] R. Ahmed, T. Wild, and F. Schaich, "Coexistence of UF-OFDM and CPOFDM," in Proc. of 2016 IEEE 83rd Vehicular Technology Conference (VTC Spring), Nanjing, May 2016, pp. 1-5.

[43] "Universal Mobile Telecommunications System (UMTS); User Equipment (UE) radio transmission and reception (FDD)," 3GPP, Tech. Rep. TS 25.101 V14.0.0, May 2017.

[44] "LTE; Evolved Universal Terrestrial Radio Access (E-UTRA); Radio Frequency (RF) system scenarios," 3GPP, Tech. Rep. TR 36.942 V14.0.0, Apr. 2017.

[45] D. Chen, D. Qu, T. Jiang, and Y. He, "Prototype Filter Optimization to Minimize Stopband Energy With NPR Constraint for Filter Bank Multicarrier Modulation Systems," IEEE Transactions on Signal Processing, vol. 61 , no. 1 , pp. 159-169, Jan. 2013

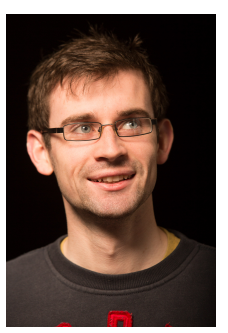

Conor Sexton is pursuing a Ph.D. on wireless communications at the CONNECT Telecommunications Research Centre, headquartered in Trinity College Dublin. He received his B.A.I. degree in Computer and Electronic Engineering from Trinity College Dublin in 2015. His research focuses on the system level implications of new PHY technologies, and providing unified visions of future networks in a manner that facilitates continuous advancement.

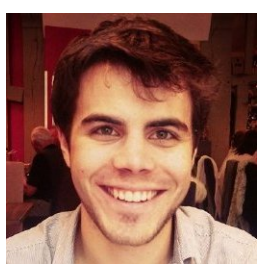

Quentin Bodinier is currently R\&D project manager at BiOSENCY, a French medtech startup. Before that, he obtained his Ph.D. on wireless communications for $5 \mathrm{G}$ in SCEE research team, at CentraleSupélec, Rennes, France, in 2017. He also obtained the Engineering Degree and M. Sc in wireless communications, embedded electronics and networks, from Supélec (now CentraleSupélec) in Rennes, France in 2014. During his Ph.D., his research activities focused on studying how new Physical layer technologies such as advanced multicarrier waveforms can enhance the coexistence of $5 \mathrm{G}$ communications, like D2D, M2M or IoT with legacy LTE networks.

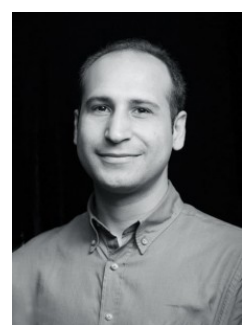

Arman Farhang received the B.Sc. degree in telecommunications engineering from the Azad University of Najafabad, Iran, in 2007, the M.Sc. degree in telecommunications engineering from the Sadjad University of Technology, Mashhad, Iran, in 2010, and the Ph.D. degree from the Trinity College Dublin, Ireland, in 2016. Since then he was a Research Fellow with the Irish National Telecommunications Research Centre (CONNECT), Trinity College Dublin, Ireland until 2018. He is currently a Lecturer with University College Dublin. His areas of research include wireless communications, digital signal processing for communications, multiuser communications, and multicarrier systems.

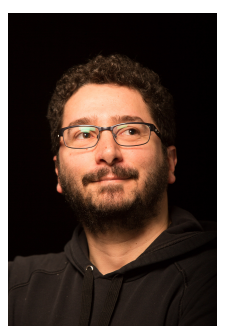

Nicola Marchetti is currently Assistant Professor in Wireless Communications at Trinity College Dublin, Ireland. He performs his research under the Trinity Information and Complexity Labs (TRICKLE) and the Irish Research Centre for Future Networks and Communications (CONNECT). He received the $\mathrm{PhD}$ in Wireless Communications from Aalborg University, Denmark in 2007, and the M.Sc. in Electronic Engineering from University of Ferrara, Italy in 2003. He also holds an M.Sc. in Mathematics which he received from Aalborg University in 2010. His collaborations include research projects in cooperation with Nokia Bell Labs and US Air Force Office of Scientific Research, among others. His research interests include Adaptive and Self-Organizing Networks, Complex Systems Science for Communication Networks, PHY Layer, Radio Resource Management. He has authored in excess of 100 journals and conference papers, 2 books and 7 book chapters, holds 2 patents, and received 4 best paper awards.

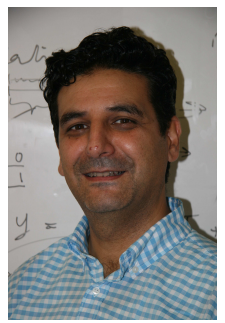

Faouzi Bader (SM07) received the Ph.D. degree (with Honours) in telecommunications from Universidad Politécnica de Madrid (UPM), Madrid, Spain, in 2002. He joined the Centre Technologic de Telecomunicacions de Catalunya (CTTC), BarcelonaSpain, as Associate Researcher, in 2002, and was nominated in 2006 as a Senior Research Associate at same institution. Since June 2013, he is as Associate Professor at École Supérieure d'Electricité-Supélec in Rennes-France, and since 2017 as honorary Adjunct Professor at University of Technology, SydneyAustralia . His research activities mainly focus on IMT-Advanced systems, advanced multicarrier waveforms, and frequency allocation techniques in relay cognitive environments. He has published over 120 papers in peerreviewed journals and international conferences, more than 13 book chapters, and edited 4 books. He served as Technical Program Committee member in major IEEE ComSoc and VTS conferences, as associate editor in Emerging Telecommunications Technologies-ETT Journal (2013-2016), and as guest editor at EURASIP Journal on Advances in Signal Processing (JASP).

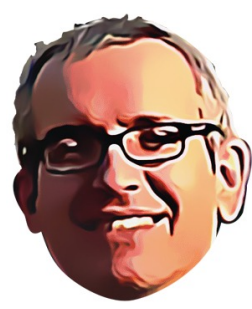

Luiz A. DaSilva holds the chair of Telecommunications at Trinity College Dublin, where he is a co-principal investigator of CONNECT, a telecommunications centre funded by the Science Foundation Ireland. Prior to joining Trinity College, Prof DaSilva was with the Bradley Department of Electrical and Computer Engineering at Virginia Tech. His research focuses on distributed and adaptive resource management in wireless networks, and in particular radio resource sharing and the application of game theory to wireless networks. Prof DaSilva is a Fellow of Trinity College Dublin, an IEEE Communications Society Distinguished Lecturer and a Fellow of the IEEE, for contributions to cognitive networks and to resource management in wireless networks. 